\title{
Pharmacotherapies to tics: a systematic review
}

Meta-Analysis

\author{
Zuojie Zhang ${ }^{1,2,3, *}$, Chunsong Yang ${ }^{1,2, *}$, Ling-Li Zhang ${ }^{1,2}$, Qiusha Yi $^{3}$, Bo Liu ${ }^{1,2}$, Jing \\ Zeng $^{1,2}$ and Dan $\mathbf{Y u}^{4}$ \\ ${ }^{1}$ Department of Pharmacy, Evidence-Based Pharmacy Center, West China Second Hospital, Sichuan University, Chengdu, China \\ ${ }^{2}$ Key Laboratory of Birth Defects and Related Diseases of Women and Children (Sichuan University), Ministry of Education, \\ Chengdu, China \\ ${ }^{3}$ West China School of Pharmacy, Sichuan University, Chengdu, China \\ ${ }^{4}$ Department of Neurology, West China Second Hospital, Sichuan University, Chengdu, China \\ * These authors have contributed equally to this work \\ Correspondence to: Ling-Li Zhang, email: zhlingli@sina.com
}

Keywords: tics; efficacy; meta-analysis; review; pharmacotherapy

Received: March 24, $2017 \quad$ Accepted: March 19, $2018 \quad$ Published: June 15, 2018

Copyright: Zhang et al. This is an open-access article distributed under the terms of the Creative Commons Attribution License 3.0 (CC BY 3.0), which permits unrestricted use, distribution, and reproduction in any medium, provided the original author and source are credited.

\section{ABSTRACT}

The efficacy of all pharmacotherapies for patients suffering from tics were unclear. Literatures were searched from Medline, Embase, The Cochrane Library, and four Chinese databases. The primary efficacy outcome scale was defined as the Yale Global Tic Severity Scale (YGTSS). Overall estimates of pooled weighted mean difference (WMD) with $95 \%$ confidence interval (CI) were calculated for each outcome measure. A total of 53 trials were included. Meta-analysis suggested that alpha-2 adrenergic agonist agents and atypical antipsychotic agents were effective in improving tics, which included the maximum number of trials. Typical antipsychotic agents were associated with severer side-effects than alpha-2 adrenergic agonist agents. Besides, Traditional Chinese Medicine showed positive effects in YGTSS (NingDong Granule: WMD=-7.100, 95\% CI, -10.430- -3.770; 5-Ling Granule: WMD =-11.300, 95\% CI, -14.208- -8.392), while glutamate modulators (D-serine, $\mathrm{N}$-Acetylcysteine and riluzole) might not be working. In summary, alpha-2 adrenergic agonist agents were associated with the optimal weigh between efficacy and safety. However, the significant factor of limited trials and sample sizes discounted these findings. Further better studies are necessary to ascertain them.

\section{INTRODUCTION}

Tourette's syndrome (TS) and chronic tic disorder (CTD) are neurodevelopmental diseases characterised by the appearance of at least one time phonic/vocal and motor tics within one year $[1,2]$. The onset age is typically 5 to 6 years old and the worst age is 10 to 12 years old [3]. Two epidemiological studies discovered that TS was diagnosed in around $0.3 \%$ to $1 \%$ of school age children $[4,5]$.

Tics are frequently comorbid with considerable diseases such as attention deficit hyperactivity disorder (ADHD), autism spectrum disorder, anxiety, depression, and obsessive compulsive disorder [6-10]. The nature damage of tics and corresponding comorbidities have significant adverse implications on quality of life and social environment [11-13]. In addition, caregivers undergo enormous burden and possibly tend to develop psychological diseases [14]. Children as well as caregivers emphasized the importance of tics reduction. So, seeking for appropriate treatments to improve tics in time is a clinical priority.

There are several types of interventions for tics including pharmacotherapies, behavioural therapies, and physical therapies, but the most widely used and mainstay treatment remains pharmacotherapy $[15,16]$. And among pharmacotherapies, antipsychotics were generally recognized. Although, several cognitive behavioural therapies, such as cognitive-behavioral approach, 
cognitive psychophysiological treatment, behavioural therapy, and habit reversal therapy, were supported to use in tics with many high-quality evidences and some of them were inserted in the Canadian guidelines for evidencebased practices $[17,18,19,20]$, surveys found that these approaches might be poor to access and difficult to use [21]. In addition, clinical trials of these treatments were impeded by several reasons including failing to blind participants and the reasonability of their use [19].

As to western medicine, none were developed to target at tics, although tics have been considered as biological conditions [22]. Despite this, these treatments have been widely applied to suppress tics, including alpha-2 adrenergic agonist agent, antipsychotic drugs, anticonvulsant, analgesic, glutamate agonist, etc. Also, many clinical trials and systemic reviews were conducted to evaluate the efficacy of pharmacotherapies for tics [23-25]. However, these studies reached mixed results. For several interventions, some evidences suggested that they were effective in decreasing disease burden, while others yielded the opposite results [26-30]. And none has summarized all pharmacotherapies. Currently, more and more powerful national groups, such as the American Academy of Child and Adolescent Psychiatry, the Canadian Academy of Child and Adolescent Psychiatry, the European Society for the Study of Tourette Syndrome, and the Chinese Medical Association, established clinical guidelines to introduce the use of pharmacotherapy for tics [21, 23]. These recommendations demonstrated the increasing focus on the use of pharmacotherapies for tics, but many first-line treatments were associated with few evidences.

Therefore, in order to improve previous research, all trials were collected to evaluate the efficacy and safety of pharmacotherapies for tics patients, in which pharmacotherapies were compared with placebo or any other pharmacotherapies.

\section{RESULTS}

\section{Study characteristics}

Fifty-three studies [26-29, 31-79] including 3155 patients were identified after literature selection, of which 15 studies were associated with mixed participants (children and adult). Types of treatments and numbers of included research were summarized in Table 1. PRISMA flowchart was displayed in Figure 1.

The characteristics of included studies were depicted in Table 2. Outcome data could not be available from 12 trials even by contacting relevant authors, so they were excluded from meta-analyses. Specific reasons were displayed as follows:1) 5 studies did not reported any of the four outcomes included in this meta-analysis $[31,34,43,53,65]$; 2) 1 study only reported whether the differences between experimental group and control group were significant, but it lacked outcome data [36]; 3)
2 studies only reported outcome data at endpoint without scores at baseline $[33,35]$; 4) 4 studies only reported mean improvement data but weighted mean differences (WMD) with the $95 \%$ confidence interval (CI) were failed to be received [42, 59-61].

\section{Risk of bias}

Risk of bias were showed in Table 3. The methodology qualities of randomisation and allocation concealment were less satisfactory. There were merely 15 reports $(28 \%)$ which clearly described the generation of random sequence and 11 reports (20.8\%) which described allocation concealment. Similarly, bindings of participant and outcome assessment were unspecified, which made unclear risk in 37 studies $(64.2 \%)$ for binding of participant and in 43 studies $(81.1 \%)$ for binding of outcome assessment. And none of the studies were judged to be prone to high risk of bias. The risk of bias regarding incomplete outcome data were high in 5 reports $(9.4 \%)$ due to high rate of dropout with little explanation and low in 26 reports (49\%). Selective report was not identified in any of the included articles. Finally, only 2 articles (3.8\%) were found to be associated with other source of bias owning to obvious unbalance of baseline.

\section{Meta-analytic results}

Forty-one studies of the included 53 studies involving 34 pharmacological interventions reported one of the outcome measures and were brought into meta-analyses, among which 33 studies (29 types of intervention) compared pharmacotherapies with placebo and 11 studies (16 types of intervention) compared pharmacotherapies with each other (3 studies were placebo controlled as well).

\section{Placebo controlled comparisons}

Table 4 to Table 7 demonstrated the efficacy of pharmacotherapies compared to placebo in each outcome measure.

Twenty-two interventions (from 33 studies) were more efficacious than placebo. It's worth noting that among the above effective treatments, only 4 interventions including guanfacine, pergolide, atomoxetine, and nicotine patch incorporated two trials, while the rest of these merely incorporated one.

\section{Alpha-2 adrenergic agonist agents}

Three studies evaluated the efficacy of alpha-2 adrenergic agonist agents. Guanfacine from two trials showed positive effect for children suffering from tics (YGTSS:WMD= -4.596, 95\% CI, -8.798- -0.393) (Figure 2). Clonidine was superior to placebo (CGI: WMD=$0.600,95 \% \mathrm{CI},-0.996--0.204)$ in one trial conducted in mixed patients. 
Table 1: Types of treatments and numbers of researches included

Intervention

Number of studies

Alpha-2 adrenergic agonist agent

Guanfacine

Clonidine

Clonidine Patch

1

Lofexidine

Analgesic

Naltrexone

Propoxyphene

Anticonvulsant

Levetiracetam

Topiramate

Antidepressant

Fluoxetine

Fluvoxamine

Desipramine

Deprenyl

Antipsychotic agent

Typical Neuroleptics

Haloperidol plus Trihexyphenidyl

Haloperidol

Pimozide

Sulpiride

Atypical Neuroleptics

Ziprasidone

Risperidone

Aripiprazole

Tiapride

Olanzapine

Cannabis

Delta-9-tetrahydrocannabinol

CNS stimulant

Methylphenidate

5

Dextroamphetamine

Methylphenidate plus Clonidine

Cholinoceptor blocking drugs

Mecamylamine

1

(Continued) 
Dopaminergic agent

$\begin{array}{ll}\text { Pergolide } & 2\end{array}$

$\begin{array}{ll}\text { Pramipexole } & 1\end{array}$

Talipexole 1

Gamma-aminobutyric acidB receptor agonist

Baclofen 1

Glutamate agonist and antagonist

D-serine 1

N-Acetylcysteine $\quad 1$

$\begin{array}{ll}\text { Riluzole } & 1\end{array}$

Selective norepinephrine reuptake inhibitor

Atomoxetine $\quad 2$

Smoking cessation agent

Nicotine patch

Smoking cessation agent plus Antipsychotic drugs

Nicotine patch plus Haloperidol

Traditional Chinese medicine

NingDong Granule

1

5-Ling Granule

1

Ningdong Granule plus Haloperidol $\quad 1$

Qufeng Zhidong Recipe $\quad 2$

5HT3-receptor antagonists

Ondansetron

Metoclopramide

1

\section{Analgesic}

Evidences of two analgesics from 2 mixed patients' studies showed opposite results. Propoxyphene was effective in suppressing tics (TSGS: WMD=-8.700, 95\% CI, -14.711- -2.689), while naltrexone failed to improve tics (TSGS: WMD=-0.100, 95\% CI, -6.4266.226).

\section{Anticonvulsants}

Similarly, evidences of two anticonvulsants showed opposite results. The trial by Jankovic and colleagues discovered that topiramate might be effective in suppressing children tics (YGTSS: WMD $=-9.290,95 \%$ CI, -16.697- -1.883). Even though the range of age in this study was 7-65, mean age was 16.5 with the majority of participants being children. For levetiracetam, it was found to be lack of efficacy in children (YGTSS: WMD $=0.050$, 95\% CI, -16.175- 16.275).

\section{Antidepressants}

Likewise, for antidepressants, desipramine (tricyclic antidepressant) was more efficacious than placebo in children (YGTSS:WMD $=-16.000$, 95\% CI,27.130- -4.870). However, Fluoxetine, another kind of antidepressant (selective serotonin reuptake inhibitor), was found to be lack of efficacy in mixed participants (YGTSS: WMD=1.100, 95\% CI, -6.325-8.525).

\section{Antipsychotic agents}

Nearly all antipsychotic agents yielded positive effect, and atypical antipsychotic agents covered more efficacious agents and outcome scales than typical antipsychotic agents. The following agents yielded positive effects: atypical antipsychotic agents (ziprasidone (YGTSS: WMD=-6.900, 95\% CI,-11.234-2.566); risperidone (YGTSS: $\mathrm{WMD}=-6.400,95 \% \mathrm{CI}$, -11.059- -1.741; CGI: WMD=-0.650, 95\% CI, -1.207- 
-0.093); aripiprazole (YGTSS: WMD $=-5.100,95 \% \mathrm{CI}$, -9.178- -1.022); tiapride (YGTSS: WMD=-11.700, 95\% CI, -15.101- -8.299)) and typical antipsychotic agent (haloperidol (TSSS: WMD=-1.700, 95\% CI, -3.006-0.394 ); pimozide (TSGS: $\mathrm{WMD}=-9.700,95 \% \mathrm{CI}$, -18.436- -0.964)), while the following agents yielded negative effects: ziprasidone in the outcome of CGI( $\mathrm{WMD}=-0.700,95 \% \mathrm{CI},-1.407-0.007)$, haloperidol in TSGS (WMD $=-6.100,95 \% \mathrm{CI},-15.361-3.161$ ), and pimozide in TSSS (WMD $=-0.400,95 \% \mathrm{CI},-1.952$ 1.152). For studies conducted in mixed population, only the separate effect of risperidone in children was found (YGTSS: WMD=-7.100, 95\% CI, -12.276- -1.924).

\section{Cannabis}

Delta-9-tetrahydrocannabinol from one trial was not significantly different from placebo in the mixed participants (YGTSS: WMD=-6.500, 95\% CI, -19.1746.174; TSGS: WMD=-6.500, 95\% CI, -15.652- 2.652).

\section{CNS (central nervous system) stimulants}

Evidences showed that methylphenidate might not exacerbate tics (YGTSS: WMD $=0.035,95 \%$ CI, -4.4424.512), no matter children receiving which kinds of therapeutic regimens, including $0.1 \mathrm{mg} / \mathrm{kg}(\mathrm{WMD}=-0.759$, $95 \% \mathrm{CI},-9.270-7.752), 0.3 \mathrm{mg} / \mathrm{kg}(\mathrm{WMD}=1.263,95 \%$ $\mathrm{CI},-7.307-9.832)$, and $0.5 \mathrm{mg} / \mathrm{kg}(\mathrm{WMD}=-0.706,95 \% \mathrm{CI}$, -9.119- 7.707). There was no sign of heterogeneity from statistical test $\left(\mathrm{I}^{2}=0 \%\right)$ among each regimen.

\section{Dopaminergic agent}

Results of the pergolide and pramipexole were inconsistent. Pergolide was superior to placebo by pooling two trials

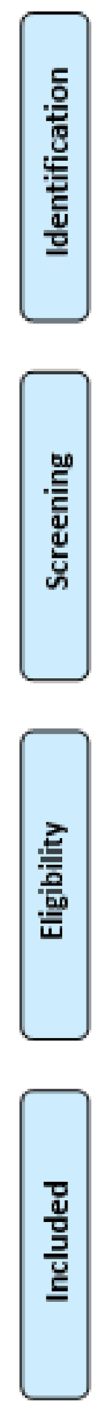

Additional records identified through other sources

$$
(n=0)
$$
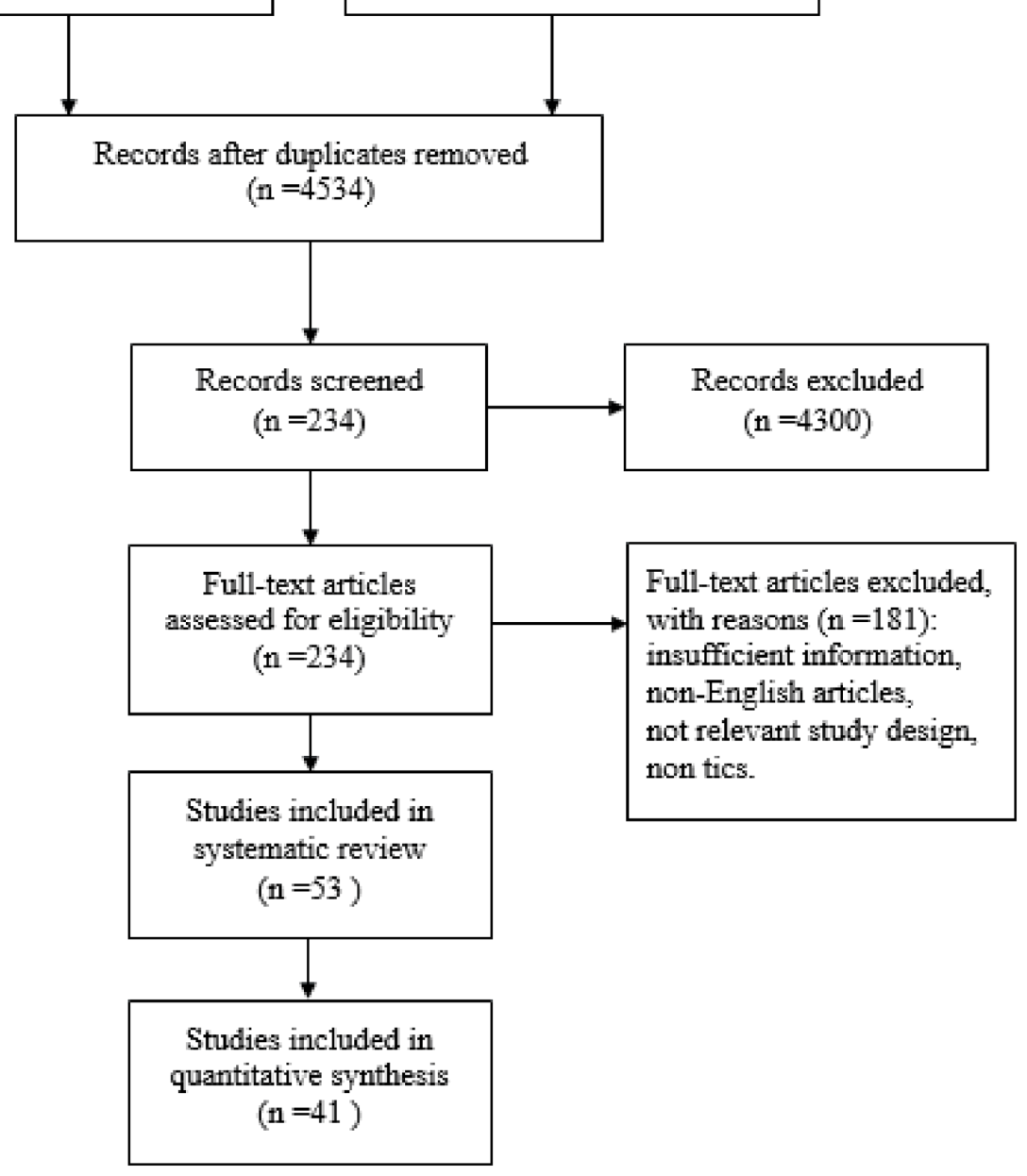

Figure 1: Flow chart of meta-analysis. 
Table 2: Characteristics of included studies

\begin{tabular}{|c|c|c|c|c|c|c|c|}
\hline References & $\begin{array}{l}\text { Diagnostic } \\
\text { criteria }{ }^{1 /} \\
\text { Indication }\end{array}$ & Treatment conditions & $\begin{array}{c}\text { Age (mean, } \\
\text { range/SD) } \\
\text { (years) }\end{array}$ & $\begin{array}{l}\text { Sample } \\
\text { (Male) }\end{array}$ & Rating Scale & $\begin{array}{c}\text { Treatment } \\
\text { duration } \\
\text { (weeks) }\end{array}$ & Design \\
\hline \multicolumn{8}{|c|}{ Alpha-2 adrenergic agonist agent } \\
\hline Scahill, 2001 & $\begin{array}{c}\mathrm{DSM} / \\
\mathrm{ADHD}+\mathrm{TD}\end{array}$ & $\begin{array}{l}\text { Placebo } \\
\text { Guanfacine }\end{array}$ & $\begin{array}{c}7-15 \\
10.4 \pm 2.01\end{array}$ & $\begin{array}{c}34(31) \\
\mathrm{C}: 17 \\
\mathrm{~T}: 17\end{array}$ & YGTSS & 8 & $\mathrm{RCT}$ \\
\hline Cummings, 2002 & DSM-IV/TS & $\begin{array}{l}\text { Placebo } \\
\text { Guanfacine }\end{array}$ & $\begin{array}{c}6-16 \\
10.4 \pm 2.3\end{array}$ & $\begin{array}{c}24(20) \\
\mathrm{C}: 12 \\
\mathrm{~T}: 12\end{array}$ & YGTSS & 4 & RCT \\
\hline Goetz, 1987 & DSM-III/TS & $\begin{array}{l}\text { Placebo } \\
\text { Clonidine }\end{array}$ & $8-62$ & $\begin{array}{c}30(23) \\
\mathrm{C}: 30 \\
\mathrm{~T}: 30\end{array}$ & NR & 12 & Crossover \\
\hline Leckman, 1991 & DSM-III/TS & $\begin{array}{l}\text { Placebo } \\
\text { Clonidine }\end{array}$ & $7-48$ & $\begin{array}{c}40(32) \\
\mathrm{C}: 19 \\
\mathrm{~T}: 21\end{array}$ & CGI & 12 & $\mathrm{RCT}$ \\
\hline Du, 2008 & CCMD-3/TD & $\begin{array}{l}\text { Placebo } \\
\text { Clonidine }\end{array}$ & $\begin{array}{c}\text { 6-18 } \\
\text { C: } 10.15 \pm 2.82 \\
\mathrm{~T}: 9.89 \pm 2.77\end{array}$ & $\begin{array}{c}437(366) \\
\mathrm{C}: 111 \\
\mathrm{~T}: 326\end{array}$ & NR & 4 & $\mathrm{RCT}$ \\
\hline Singer, 1994 & $\begin{array}{l}\text { DSM-III-R/ } \\
\text { TS+ADHD }\end{array}$ & $\begin{array}{c}\text { Placebo } \\
\text { Clonidine, } \\
\text { Desipramine }\end{array}$ & $2-11$ & $\begin{array}{c}34(31) \\
\mathrm{C}: 34 \\
\mathrm{~T} 1: 34 \\
\mathrm{~T} 2: 34\end{array}$ & NR & 6 & $\mathrm{RCT}$ \\
\hline Zhong, 2007 & CCMD-III/TD & $\begin{array}{l}\text { Placebo patch } \\
\text { Clonidine patch }\end{array}$ & $6-18$ & $\begin{array}{l}76(49) \\
\mathrm{C}: 18 \\
\mathrm{~T}: 58\end{array}$ & NR & 4 & Parallel RCT \\
\hline Niederhofer, 2003 & $\begin{array}{c}\text { DSM-IV/ } \\
\text { ADHD+TD }\end{array}$ & $\begin{array}{l}\text { Placebo } \\
\text { Lofexidine }\end{array}$ & $\begin{array}{c}7-15 \\
10.4 \pm 2.01\end{array}$ & $\begin{array}{l}44(41) \\
\mathrm{C}: 22 \\
\mathrm{~T}: 22\end{array}$ & NR & 8 & RCT \\
\hline Hedderick, 2009 & $\mathrm{DSM} / \mathrm{TS}$ & $\begin{array}{l}\text { Levetiracetam } \\
\text { Clonidine }\end{array}$ & $8-30$ & $\begin{array}{l}10(7) \\
\mathrm{C}: 10 \\
\mathrm{~T}: 10\end{array}$ & $\begin{array}{c}\text { YGTSS } \\
\text { CGI }\end{array}$ & 15 & Crossover \\
\hline \multicolumn{8}{|l|}{ Analgesic } \\
\hline Kurlan, 1991 & DSM-III-R/TS & $\begin{array}{c}\text { Placebo } \\
\text { Naltrexone, } \\
\text { Propoxyphene }\end{array}$ & $33 \pm 10$ & $\begin{array}{c}10(8) \\
\text { C:3 } \\
\text { T1:3 } \\
\text { T2: } 4\end{array}$ & TSGS & 6 & $\mathrm{RCT}$ \\
\hline \multicolumn{8}{|l|}{ Anticonvulsant } \\
\hline Smith-hicks, 2007 & DSM-IV/TS & $\begin{array}{c}\text { Placebo } \\
\text { Levetiracetam }\end{array}$ & $\begin{array}{c}8-16 \\
12.2 \pm 2.3\end{array}$ & $\begin{array}{c}22(21) \\
\mathrm{C}: 22 \\
\mathrm{~T}: 20\end{array}$ & $\begin{array}{c}\text { YGTSS } \\
\text { CGI }\end{array}$ & 10 & Crossover \\
\hline Jankovic, 2010 & DSM-IV/TS & $\begin{array}{l}\text { Placebo } \\
\text { Topiramate }\end{array}$ & $\begin{array}{c}7-65 \\
\mathrm{C}: 14.1 \pm 8.35 \\
\mathrm{~T}: 18.8 \pm 10.93\end{array}$ & $\begin{array}{l}29(26) \\
\mathrm{T}: 15 \\
\mathrm{C}: 14\end{array}$ & YGTSS & 10 & RCT \\
\hline \multicolumn{8}{|l|}{ Antidepressant } \\
\hline Scahill, 1997 & DSM-III-R/TS & $\begin{array}{l}\text { Placebo } \\
\text { Fluoxetine }\end{array}$ & $8-33$ & $\begin{array}{l}14(9) \\
\mathrm{C}: 10 \\
\mathrm{~T}: 10\end{array}$ & YGTSS & 20 & Crossover \\
\hline George, 1993 & DSM-III-R/TS & $\begin{array}{c}\text { Sulpiride } \\
\text { Fluvoxamine }\end{array}$ & $\begin{array}{l}\mathrm{C}: 29.6 \pm 2.9 \\
\mathrm{~T}: 28.3 \pm 3.2\end{array}$ & $\begin{array}{l}11(8) \\
\mathrm{C}: 11 \\
\mathrm{~T}: 11\end{array}$ & YGTSS & 18 & Crossover \\
\hline Spencer, 2002 & $\begin{array}{l}\text { DSM-IV-R/ } \\
\text { ADHD+TD }\end{array}$ & $\begin{array}{c}\text { Placebo } \\
\text { Desipramine }\end{array}$ & $\begin{array}{c}5-17 \\
\mathrm{C}: 11.3 \pm 3.0 \\
\mathrm{~T}: 10.6 \pm 2.4\end{array}$ & $\begin{array}{l}41(34) \\
\mathrm{C}: 20 \\
\mathrm{~T}: 21\end{array}$ & YGTSS & 6 & $\mathrm{RCT}$ \\
\hline
\end{tabular}

(Continued) 


\begin{tabular}{|c|c|c|c|c|c|c|c|}
\hline References & $\begin{array}{l}\text { Diagnostic } \\
\text { criteria }^{1 /} \\
\text { Indication }\end{array}$ & Treatment conditions & $\begin{array}{c}\text { Age (mean, } \\
\text { range/SD) } \\
\text { (years) }\end{array}$ & $\begin{array}{l}\text { Sample } \\
\text { (Male) }\end{array}$ & Rating Scale & $\begin{array}{c}\text { Treatment } \\
\text { duration } \\
\text { (weeks) }\end{array}$ & Design \\
\hline Feigin, 1995 & $\begin{array}{l}\text { DSM-IIIR/ } \\
\text { ADHD+TS }\end{array}$ & $\begin{array}{l}\text { Placebo } \\
\text { Deprenyl }\end{array}$ & $7-16$ & $\begin{array}{c}24(21) \\
\mathrm{T}: 24 \\
\mathrm{C}: 24\end{array}$ & YGTSS & 8 & Crossover \\
\hline \multicolumn{8}{|c|}{ Antipsychotic agent } \\
\hline Shapiro, 1989 & DSM-III/TS & $\begin{array}{c}\text { Placebo } \\
\text { Haloperidol, Pimozide }\end{array}$ & $\begin{array}{c}8-65 \\
21.1 \pm 11.0\end{array}$ & $\begin{array}{c}57(44) \\
\text { C:19 } \\
\text { T1:18 } \\
\text { T2:20 }\end{array}$ & TSSS & 6 & Crossover \\
\hline Sallee, 1997 & DSM-III-R/TS & $\begin{array}{c}\text { Placebo } \\
\text { Haloperidol, Pimozide }\end{array}$ & $\begin{array}{c}7-16 \\
10.2 \pm 2.5\end{array}$ & $\begin{array}{c}22(17) \\
\mathrm{C}: 22 \\
\mathrm{~T} 1: 22 \\
\mathrm{~T} 2: 22\end{array}$ & TSGS & 24 & Crossover \\
\hline Shapirc, 1984 & DSM-III/TS & $\begin{array}{l}\text { Placebo } \\
\text { Pimozide }\end{array}$ & $\begin{array}{c}11-53 \\
24.65 \pm 2.71\end{array}$ & $\begin{array}{c}20(13) \\
\mathrm{C}: 20 \\
\mathrm{~T}: 20\end{array}$ & NR & 14 & Crossover \\
\hline Sallee, 1999 & DSM-IV/TS & $\begin{array}{l}\text { Placebo } \\
\text { Ziprasidone }\end{array}$ & $\begin{array}{c}7-16 \\
\mathrm{C}: 11.3(7-14) \\
\mathrm{T}: 11.8(8-16)\end{array}$ & $\begin{array}{c}28(22) \\
\mathrm{C}: 12 \\
\mathrm{~T}: 16\end{array}$ & $\begin{array}{l}\text { YGTSS } \\
\text { CGI }\end{array}$ & 8 & $\mathrm{RCT}$ \\
\hline Scahill, 2003 & DSM-IV/TS & $\begin{array}{l}\text { Placebo } \\
\text { Risperidone }\end{array}$ & $\begin{array}{c}6-62 \\
19.7 \pm 17.01\end{array}$ & $\begin{array}{c}34(30) \\
\mathrm{C}: 18 \\
\mathrm{~T}: 16\end{array}$ & YGTSS & 8 & $\mathrm{RCT}$ \\
\hline Dion, 2002 & DSM-IV-R/TS & $\begin{array}{l}\text { Placebo } \\
\text { Risperidone }\end{array}$ & $\begin{array}{c}14-49 \\
\mathrm{C}: 31(17-49) \\
\mathrm{T}: 33(14-45)\end{array}$ & $\begin{array}{c}46(36) \\
\mathrm{C}: 23 \\
\mathrm{~T}: 23\end{array}$ & $\begin{array}{l}\text { CGI } \\
\text { TSSS }\end{array}$ & 8 & $\mathrm{RCT}$ \\
\hline Gaffney, 2002 & DSM-III-R/TS & $\begin{array}{l}\text { Clonidine } \\
\text { Risperidone }\end{array}$ & $\begin{array}{c}7-17 \\
\mathrm{~T}: 10.4 \pm 2.7 \\
\mathrm{C}: 12.1 \pm 3.0\end{array}$ & $\begin{array}{c}21(19) \\
\mathrm{C}: 12 \\
\mathrm{~T}: 9\end{array}$ & YGTSS & 8 & $\mathrm{RCT}$ \\
\hline Gilbert, 2004 & DSM-IV-TR/TD & $\begin{array}{l}\text { Pimozide } \\
\text { Risperidone }\end{array}$ & $\begin{array}{c}7-17 \\
11 \pm 2.5\end{array}$ & $\begin{array}{c}19(15) \\
\mathrm{C}: 18 \\
\mathrm{~T}: 18\end{array}$ & $\begin{array}{l}\text { YGTSS } \\
\text { CGI }\end{array}$ & 4 & Crossover \\
\hline Yoo, 2013 & DSM-IV/TD & $\begin{array}{c}\text { Placebo } \\
\text { Aripiprazole }\end{array}$ & $\begin{array}{c}6-18 \\
\text { C: } 11.0 \pm 25 \\
\text { T:10.9 } \pm 3.0\end{array}$ & $\begin{array}{c}61(53) \\
\mathrm{C}: 29 \\
\mathrm{~T}: 32\end{array}$ & YGTSS & 10 & $\mathrm{RCT}$ \\
\hline Ghanizadeh, 2014 & DSM-IV/TD & $\begin{array}{l}\text { Risperidone } \\
\text { Aripiprazole }\end{array}$ & $6-18$ & $\begin{array}{c}60 \\
\mathrm{C}: 29 \\
\mathrm{~T}: 31\end{array}$ & YGTSS & 8 & $\mathrm{RCT}$ \\
\hline Eggers, 1988 & ICD-10/TS & $\begin{array}{l}\text { Placebo } \\
\text { Tiapride }\end{array}$ & $7-18$ & $\begin{array}{c}17(25) \\
\mathrm{C}: 17 \\
\mathrm{~T}: 17\end{array}$ & NR & 26 & Crossover \\
\hline Onofrj, 2000 & Unclear/TS & $\begin{array}{l}\text { Pimozide } \\
\text { Olanzapine }\end{array}$ & $19-40$ & $\begin{array}{l}4(4) \\
\mathrm{C} 1: 4 \\
\mathrm{~T} 1: 4\end{array}$ & TSGS & 52 & Crossover \\
\hline \multicolumn{8}{|l|}{ Cannabis } \\
\hline Muller-vahl,2002 & DSM-III R/TS & $\begin{array}{l}\text { Placebo } \\
\text { Delta 9-tetrahy- } \\
\text { drocannabinol }\end{array}$ & $18-66$ & $\begin{array}{c}12(11) \\
\text { C:6 } \\
\mathrm{T}: 6\end{array}$ & $\begin{array}{c}\text { YGTSS } \\
\text { TSGS }\end{array}$ & 4 & $\mathrm{RCT}$ \\
\hline \multicolumn{8}{|l|}{ CNS stimulant } \\
\hline Gadow, 1995 & $\begin{array}{l}\text { DSM-III-R/ } \\
\text { ADHA+TD }\end{array}$ & $\begin{array}{c}\text { Placebo } \\
\text { Methylphenidate }\end{array}$ & 810 month & $\begin{array}{c}34(31) \\
\text { C:8 } \\
\text { T1:9 } \\
\text { T2:8 } \\
\text { T3:9 }\end{array}$ & YGTSS & 2 & $\mathrm{RCT}$ \\
\hline Gadow, 1999 & $\begin{array}{l}\text { DSM-III-R/ } \\
\text { ADHD+TS }\end{array}$ & $\begin{array}{c}\text { Placebo } \\
\text { Methylphenidate }\end{array}$ & $6.1-11.9$ & $\begin{array}{c}34(31) \\
\mathrm{C}: 17 \\
\mathrm{~T}: 17\end{array}$ & $\begin{array}{l}\text { YGTSS } \\
\text { CGI }\end{array}$ & 2 & $\begin{array}{c}\mathrm{RCT} \\
\text { (Contin }\end{array}$ \\
\hline
\end{tabular}




\begin{tabular}{|c|c|c|c|c|c|c|c|}
\hline References & $\begin{array}{l}\text { Diagnostic } \\
\text { criteria }^{1 /} \\
\text { Indication }\end{array}$ & Treatment conditions & $\begin{array}{l}\text { Age (mean, } \\
\text { range/SD) } \\
\text { (years) }\end{array}$ & $\begin{array}{l}\text { Sample } \\
\text { (Male) }\end{array}$ & Rating Scale & $\begin{array}{c}\text { Treatment } \\
\text { duration } \\
\text { (weeks) }\end{array}$ & Design \\
\hline Gadow, 2007 & $\begin{array}{c}\text { DSM/ } \\
\text { ADHA+TD }\end{array}$ & $\begin{array}{c}\text { Placebo } \\
\text { Methylphenidate }\end{array}$ & $\begin{array}{c}6-12 \\
8.95 \pm 1.4\end{array}$ & $\begin{array}{l}71(57) \\
\text { C:17 } \\
\text { T1:17 } \\
\text { T2:18 } \\
\text { T3:18 }\end{array}$ & YGTSS & 2 & RCT \\
\hline Castellanos, 1997 & $\begin{array}{l}\text { DSM-III-R/ } \\
\text { ADHD+TS }\end{array}$ & $\begin{array}{c}\text { Placebo } \\
\text { Methylphenidate, } \\
\text { Dextroamphetamine }\end{array}$ & $9.4 \pm 2.0$ & $\begin{array}{c}12 \\
\mathrm{C}: 10 \\
\mathrm{~T} 1: 10 \\
\mathrm{~T} 2: 10\end{array}$ & YGTSS & 9 & Crossover \\
\hline Kurlan 2002 & $\begin{array}{l}\text { DSM-IV/ } \\
\text { ADHD+ } \\
\text { TS }\end{array}$ & $\begin{array}{c}\text { Placebo } \\
\text { Methylphenidate, } \\
\text { Clonidine, } \\
\text { Methylphenidate + } \\
\text { Clonidine }\end{array}$ & $7-14$ & $\begin{array}{c}136(116) \\
\text { T1:37 } \\
\text { T2:34 } \\
\text { T3:33 } \\
\text { C:32 }\end{array}$ & YGTSS & 16 & $\mathrm{RCT}$ \\
\hline \multicolumn{8}{|c|}{ Cholinoceptor blocking drugs } \\
\hline Silver, 2001 a & DSM-IV/TS & $\begin{array}{c}\text { Placebo } \\
\text { Mecamylamine }\end{array}$ & $8-17$ & $\begin{array}{l}61(55) \\
\mathrm{C}: 32 \\
\mathrm{~T}: 29\end{array}$ & NR & 8 & $\mathrm{RCT}$ \\
\hline \multicolumn{8}{|c|}{ Dopaminergic agent } \\
\hline Gilbert, 2003 & $\begin{array}{l}\text { DSM/TS, CTDs } \\
\text { and ADHD }\end{array}$ & $\begin{array}{l}\text { Placebo } \\
\text { Pergolide }\end{array}$ & $\begin{array}{c}7-17 \\
\mathrm{C}: 10.4 \pm 2.2 \\
\mathrm{~T}: 11.5 \pm .9\end{array}$ & $\begin{array}{l}51(77) \\
\mathrm{C}: 15 \\
\mathrm{~T}: 36\end{array}$ & YGTSS & 8 & $\mathrm{RCT}$ \\
\hline Gilbert, 2000 & DSM-IV/TS & $\begin{array}{l}\text { Placebo } \\
\text { Pergolide }\end{array}$ & $7-17$ & $\begin{array}{c}24(15) \\
\mathrm{C}: 19 \\
\mathrm{~T}: 19\end{array}$ & YGTSS & 16 & Crossover \\
\hline Kurlan,2012 & DSM-IV/TS & $\begin{array}{c}\text { Placebo } \\
\text { Pramipexole }\end{array}$ & $6-17$ & $\begin{array}{c}63 \\
\mathrm{C}: 20 \\
\mathrm{~T}: 43\end{array}$ & YGTSS & 6 & $\mathrm{RCT}$ \\
\hline Goetz,1994 & DSM-III-R/TS & $\begin{array}{l}\text { Placebo } \\
\text { Talipexole }\end{array}$ & $19-63$ & $\begin{array}{c}13(13) \\
\mathrm{C}: 8 \\
\mathrm{~T}: 8\end{array}$ & NR & 24 & Crossover \\
\hline \multicolumn{8}{|c|}{ Gamma-aminobutyric acid-B receptor agonist } \\
\hline Singer, 2001 & DSM-IV/TS & $\begin{array}{l}\text { Placebo } \\
\text { Baclofen }\end{array}$ & $8-14$ & $\begin{array}{l}10(7) \\
\mathrm{C}: 10 \\
\mathrm{~T}: 9\end{array}$ & CGI & 10 & $\mathrm{RCT}$ \\
\hline \multicolumn{8}{|c|}{ Glutamate agonist and antagonist } \\
\hline Lemmon, 2015 & $\begin{array}{l}\text { TS was defined } \\
\text { by the TS } \\
\text { Classification } \\
\text { Study Group }\end{array}$ & $\begin{array}{c}\text { Placebo } \\
\text { D-serine, Riluzole }\end{array}$ & $9-18$ & $\begin{array}{l}24(21) \\
\text { C:5 } \\
\text { T1:10 } \\
\text { T2:9 }\end{array}$ & YGTSS & 8 & $\mathrm{RCT}$ \\
\hline Bloch, 2016 & $\begin{array}{l}\text { DSM-IV/ } \\
\text { TS+TCD }\end{array}$ & $\begin{array}{c}\text { Placebo } \\
\text { N-Acetylcysteine }\end{array}$ & $8-17$ & $\begin{array}{c}31(24) \\
\mathrm{C}: 14 \\
\mathrm{~T}: 17\end{array}$ & $\begin{array}{l}\text { YGTSS } \\
\text { CGI }\end{array}$ & 12 & $\mathrm{RCT}$ \\
\hline \multicolumn{8}{|c|}{ Selective norepinephrine reuptake inhibitor } \\
\hline Allen, 2005 & $\begin{array}{l}\text { DSM-IV/ } \\
\text { ADHD+TD }\end{array}$ & $\begin{array}{c}\text { Placebo } \\
\text { Atomoxetine }\end{array}$ & $\begin{array}{c}7-17 \\
11.2 \pm 2.5\end{array}$ & $\begin{array}{c}148(131) \\
\mathrm{C}: 76 \\
\mathrm{~T}: 72\end{array}$ & $\begin{array}{l}\text { YGTSS } \\
\text { CGI }\end{array}$ & 18 & $\mathrm{RCT}$ \\
\hline Spencer, 2008 & $\begin{array}{c}\text { DSM-IV/ } \\
\text { ADHD+ TS }\end{array}$ & $\begin{array}{c}\text { Placebo } \\
\text { Atomoxetine }\end{array}$ & $\begin{array}{c}7-17 \\
11.2 \pm 2.4\end{array}$ & $\begin{array}{c}117(102) \\
\text { C:56 } \\
\text { T:61 }\end{array}$ & $\begin{array}{l}\text { YGTSS } \\
\text { CGI }\end{array}$ & 18 & $\mathrm{RCT}$ \\
\hline
\end{tabular}

(Continued) 


\begin{tabular}{|c|c|c|c|c|c|c|c|}
\hline References & $\begin{array}{l}\text { Diagnostic } \\
\text { criteria }^{1 /} \\
\text { Indication }\end{array}$ & Treatment conditions & $\begin{array}{l}\text { Age (mean, } \\
\text { range/SD) } \\
\text { (years) }\end{array}$ & $\begin{array}{l}\text { Sample } \\
\text { (Male) }\end{array}$ & Rating Scale & $\begin{array}{c}\text { Treatment } \\
\text { duration } \\
\text { (weeks) }\end{array}$ & Design \\
\hline \multicolumn{8}{|c|}{ Smoking cessation agent } \\
\hline Silver, $2001 \mathrm{~b}$ & DSM-IV/TD & $\begin{array}{c}\text { Placebo } \\
\text { Nicotine patch }\end{array}$ & $\begin{array}{l}\mathrm{T}: 10.5 \pm 1.8 \\
\mathrm{C}: 11.7 \pm 2.6\end{array}$ & $\begin{array}{l}70(63) \\
\mathrm{C}: 35 \\
\mathrm{~T}: 35\end{array}$ & YGTSS & 4.71 & $\mathrm{RCT}$ \\
\hline \multicolumn{8}{|c|}{ Smoking cessation agent plus Antipsychotic drugs } \\
\hline Mcconville 1992 & DSM-III-R/TS & $\begin{array}{c}\text { Placebo } \\
\text { Nicotine patch plus } \\
\text { haloperidol, Nicotine } \\
\text { patch }\end{array}$ & $8-46$ & $\begin{array}{l}19(16) \\
\text { C:5 } \\
\text { T1:10 } \\
\text { T2:9 }\end{array}$ & $\begin{array}{l}\text { YGTSS } \\
\text { CGI }\end{array}$ & NR & $\mathrm{RCT}$ \\
\hline \multicolumn{8}{|c|}{ Traditional Chinese Medicine } \\
\hline Zhao, 2010 & DSM-IV-TR/TS & $\begin{array}{c}\text { Placebo } \\
\text { Ningdong Granule }\end{array}$ & $\begin{array}{c}7-18 \\
\mathrm{C}: 12.50 \pm 2.87 \\
\mathrm{~T}: 11.95 \pm 2.93\end{array}$ & $\begin{array}{c}64(57) \\
\mathrm{C}: 31 \\
\mathrm{~T}: 33\end{array}$ & YGTSS & 8 & RCT \\
\hline Zheng, 2016 & DSM-IV/TS & $\begin{array}{l}\text { Placebo } \\
\text { Tiapride, 5-Ling } \\
\text { Granule }\end{array}$ & $\begin{array}{c}5-18 \\
9.8 \pm 3.0\end{array}$ & $\begin{array}{l}603(511) \\
\text { C:117 } \\
\text { T1:123 } \\
\text { T2:363 }\end{array}$ & YGTSS & 8 & $\mathrm{RCT}$ \\
\hline Li, 2009 & DSM-IV/TS & $\begin{array}{c}\text { Haloperidol } \\
\text { Ningdong Granule plus } \\
\text { Haloperidol }\end{array}$ & $\begin{array}{l}\text { C:9.60 } \pm 2.95 \\
\mathrm{~T}: 9.59 \pm 3.00\end{array}$ & $\begin{array}{c}90(70) \\
\mathrm{C}: 30 \\
\mathrm{~T}: 60\end{array}$ & YGTSS & 25.71 & RCT \\
\hline $\mathrm{Wu}, 2010$ & $\begin{array}{c}\text { ICD-10, TCM/ } \\
\text { TD }\end{array}$ & $\begin{array}{l}\text { Haloperidol plus } \\
\text { Trihexyphenidyl. } \\
\text { Qufeng Zhidong } \\
\text { Recipe }\end{array}$ & $\begin{array}{l}\text { C: } 6.93 \pm 2.64 \\
\text { T:7.11 } \pm 3.67\end{array}$ & $\begin{array}{c}61(51) \\
\mathrm{C}: 30 \\
\mathrm{~T}: 31\end{array}$ & YGTSS & 24 & $\mathrm{RCT}$ \\
\hline Wu, 2009 & $\begin{array}{l}\text { ICD-10, DSM- } \\
\text { IV/TD }\end{array}$ & $\begin{array}{l}\text { Haloperidol plus } \\
\text { Trihexyphenidyl } \\
\text { Qufeng Zhidong } \\
\text { Recipe }\end{array}$ & $\begin{array}{l}\text { C: } 9.10 \pm 1.13 \\
\mathrm{~T}: 9.70 \pm 2.01\end{array}$ & $\begin{array}{c}81(66) \\
\mathrm{C}: 40 \\
\mathrm{~T}: 41\end{array}$ & YGTSS & 24 & $\mathrm{RCT}$ \\
\hline \multicolumn{8}{|c|}{ 5HT3-receptor antagonists } \\
\hline Toran, 2005 & DSM-IV/TS & $\begin{array}{c}\text { Placebo } \\
\text { Ondansetron }\end{array}$ & $\begin{array}{c}12-46 \\
21.7 \pm 9.14\end{array}$ & $\begin{array}{l}30(20) \\
\mathrm{C}: 15 \\
\mathrm{~T}: 15\end{array}$ & $\begin{array}{l}\text { YGTSS } \\
\text { TSGS }\end{array}$ & 3 & RCT \\
\hline Nicolson, 2005 & DSM-IV-TR/TS & $\begin{array}{c}\text { Placebo } \\
\text { Metoclopramide }\end{array}$ & $\begin{array}{c}7-18 \\
\mathrm{~T}: 12.4 \pm 2.1 \\
\mathrm{C}: 11.4 \pm 3.1\end{array}$ & $\begin{array}{c}27 \\
\mathrm{C}: 13 \\
\mathrm{~T}: 14\end{array}$ & $\begin{array}{l}\text { YGTSS } \\
\text { CGI }\end{array}$ & 8 & $\mathrm{RCT}$ \\
\hline
\end{tabular}

Note:

1. Diagnose:

DSM-IV: Diagnostic and Statistical Manual of Mental Disorders-IV

ICD-10: The International Statistical Classification of Diseases and Related Health Problems 10th Revision

DSM-III R: Diagnostic and Statistical Manual of Mental Disorders-III revision

The definition of TS Classification Study Group: onset before 18 years, multiple involuntary motor tics, one or more vocal tics, a waxing and waning course, the gradual replacement of old symptoms with new ones, the presence of tics for more than one year, the absence of other medical explanations for tics, and the observation of tics by a reliable examiner ADHD: attention deficit hyperactivity disorder

(YGTSS: WMD=-13.167, 95\% CI, -20.553- -5.781)

(Figure 3), while pramipexole was found to be lack of efficacy (YGTSS: WMD=:-0.150, 95\% CI, -2.277- 1.977).

\section{Gamma-aminobutyric acidB receptor agonist}

Baclofen was superior to placebo from one trial (CGI: WMD=-0.900, 95\% CI, -1.497- -0.303).

\section{Glutamate agonist and antagonist}

As to glutamate modulators (D-serine, $\mathrm{N}$-Acetylcysteine and riluzole), each in one trial, they were all not significantly different from placebo in the measure of YGTSS (D-serine: WMD $=-2.600,95 \% \mathrm{CI},-19.985$ 14.785; N-Acetylcysteine: $\mathrm{WMD}=2.200,95 \% \mathrm{CI},-2.830$ 7.230; riluzole $\mathrm{WMD}=-4.100,95 \% \mathrm{CI},-23.452-15.252$ ). 
Table 3: Risk of bias in studies

\begin{tabular}{|c|c|c|c|c|c|c|c|}
\hline Study ID & $\begin{array}{c}\text { Random } \\
\text { sequence } \\
\text { generation }\end{array}$ & $\begin{array}{c}\text { Allocation } \\
\text { concealment }\end{array}$ & $\begin{array}{c}\text { Blinding of } \\
\text { participants }\end{array}$ & $\begin{array}{c}\text { Blinding } \\
\text { of outcome } \\
\text { assessment }\end{array}$ & $\begin{array}{c}\text { Incomplete } \\
\text { outcome } \\
\text { data }\end{array}$ & $\begin{array}{l}\text { Selective } \\
\text { reporting }\end{array}$ & $\begin{array}{c}\text { Other } \\
\text { sources of } \\
\text { bias } \\
\end{array}$ \\
\hline Scahill, 2001 & $\mathrm{U}$ & $\mathrm{U}$ & $\mathrm{L}$ & $\mathrm{U}$ & $\mathrm{U}$ & $\mathrm{U}$ & $\mathrm{L}$ \\
\hline Cummings, 2002 & $\mathrm{U}$ & $\mathrm{L}$ & $\mathrm{L}$ & $\mathrm{U}$ & $\mathrm{U}$ & $\mathrm{U}$ & $\mathrm{L}$ \\
\hline Goetz,1987 & $\mathrm{U}$ & $\mathrm{U}$ & $\mathrm{U}$ & $\mathrm{U}$ & $\mathrm{L}$ & $\mathrm{U}$ & $\mathrm{U}$ \\
\hline Leckman,1991 & $\mathrm{U}$ & $\mathrm{U}$ & $\mathrm{U}$ & $\mathrm{U}$ & $\mathrm{H}$ & $\mathrm{U}$ & $\mathrm{L}$ \\
\hline $\mathrm{Du}, 2008$ & $\mathrm{~L}$ & $\mathrm{U}$ & $\mathrm{L}$ & $\mathrm{L}$ & $\mathrm{U}$ & $\mathrm{U}$ & $\mathrm{L}$ \\
\hline Singer,1994 & $\mathrm{U}$ & $\mathrm{U}$ & $\mathrm{U}$ & $\mathrm{U}$ & $\mathrm{L}$ & $\mathrm{U}$ & $\mathrm{U}$ \\
\hline Zhong,2007 & $\mathrm{L}$ & $\mathrm{U}$ & $\mathrm{U}$ & $\mathrm{L}$ & U & U & $\mathrm{L}$ \\
\hline Niederhofer,2003 & $\mathrm{U}$ & $\mathrm{U}$ & U & $\mathrm{L}$ & $\mathrm{U}$ & $\mathrm{U}$ & U \\
\hline Hedderick, 2009 & $\mathrm{U}$ & $\mathrm{U}$ & $\mathrm{L}$ & $\mathrm{L}$ & $\mathrm{L}$ & $\mathrm{U}$ & $\mathrm{L}$ \\
\hline Kurlan,1991 & $\mathrm{U}$ & $\mathrm{U}$ & $\mathrm{U}$ & U & $\mathrm{U}$ & U & U \\
\hline Smith-hicks, 2007 & $\mathrm{~L}$ & $\mathrm{U}$ & $\mathrm{U}$ & $\mathrm{U}$ & $\mathrm{L}$ & $\mathrm{U}$ & $\mathrm{U}$ \\
\hline Jankovic, 2010 & $\mathrm{~L}$ & $\mathrm{U}$ & $\mathrm{U}$ & $\mathrm{U}$ & $\mathrm{L}$ & $\mathrm{U}$ & $\mathrm{U}$ \\
\hline Scahill,1997 & $\mathrm{U}$ & $\mathrm{U}$ & $\mathrm{L}$ & $\mathrm{U}$ & $\mathrm{L}$ & $\mathrm{U}$ & $\mathrm{L}$ \\
\hline George, 1993 & $\mathrm{U}$ & $\mathrm{U}$ & $\mathrm{U}$ & $\mathrm{L}$ & $\mathrm{L}$ & U & $\mathrm{U}$ \\
\hline Spencer,2002 & $\mathrm{U}$ & $\mathrm{L}$ & $\mathrm{U}$ & $\mathrm{U}$ & $\mathrm{U}$ & $\mathrm{U}$ & $\mathrm{L}$ \\
\hline Feigin, 1995 & $\mathrm{~L}$ & $\mathrm{U}$ & $\mathrm{U}$ & $\mathrm{U}$ & U & U & $\mathrm{L}$ \\
\hline Shapiro, 1989 & $\mathrm{U}$ & $\mathrm{U}$ & $\mathrm{U}$ & $\mathrm{U}$ & $\mathrm{L}$ & $\mathrm{L}$ & U \\
\hline Sallee, 1997 & $\mathrm{U}$ & $\mathrm{L}$ & U & $\mathrm{U}$ & $\mathrm{L}$ & $\mathrm{U}$ & $\mathrm{U}$ \\
\hline Shapirc, 1984 & $\mathrm{U}$ & $\mathrm{U}$ & U & U & $\mathrm{L}$ & $\mathrm{L}$ & U \\
\hline Sallee, 1999 & $\mathrm{U}$ & $\mathrm{U}$ & U & $\mathrm{U}$ & U & $\mathrm{H}$ & $\mathrm{U}$ \\
\hline Scahill, 2003 & $\mathrm{~L}$ & $\mathrm{~L}$ & U & U & $\mathrm{L}$ & U & U \\
\hline Dion, 2002 & $\mathrm{U}$ & $\mathrm{U}$ & $\mathrm{U}$ & $\mathrm{U}$ & $\mathrm{L}$ & $\mathrm{L}$ & $\mathrm{U}$ \\
\hline Gaffney,2002 & $\mathrm{U}$ & $\mathrm{U}$ & $\mathrm{U}$ & $\mathrm{U}$ & $\mathrm{L}$ & $\mathrm{L}$ & $\mathrm{U}$ \\
\hline Gilbert,2004 & $\mathrm{L}$ & $\mathrm{L}$ & $\mathrm{L}$ & $\mathrm{L}$ & $\mathrm{L}$ & $\mathrm{L}$ & $\mathrm{U}$ \\
\hline Yoo,2013 & $\mathrm{U}$ & U & U & U & $\mathrm{L}$ & U & $\mathrm{L}$ \\
\hline Ghanizadeh,2014 & $\mathrm{L}$ & $\mathrm{U}$ & $\mathrm{L}$ & $\mathrm{L}$ & $\mathrm{L}$ & $\mathrm{U}$ & $\mathrm{L}$ \\
\hline Eggers, 1988 & U & $\mathrm{U}$ & $\mathrm{U}$ & U & $\mathrm{U}$ & $\mathrm{U}$ & $\mathrm{L}$ \\
\hline Onofrj, 2000 & $\mathrm{~L}$ & $\mathrm{U}$ & U & U & $\mathrm{L}$ & $\mathrm{L}$ & U \\
\hline Muller-vahl,2002 & $\mathrm{U}$ & $\mathrm{L}$ & $\mathrm{L}$ & L & $\mathrm{L}$ & $\mathrm{U}$ & $\mathrm{U}$ \\
\hline Gadow,1995 & $\mathrm{U}$ & $\mathrm{U}$ & U & U & U & U & $\mathrm{L}$ \\
\hline Gadow,1999 & $\mathrm{U}$ & $\mathrm{U}$ & $\mathrm{U}$ & $\mathrm{U}$ & $\mathrm{U}$ & $\mathrm{U}$ & $\mathrm{L}$ \\
\hline Gadow,2007 & $\mathrm{U}$ & $\mathrm{L}$ & $\mathrm{U}$ & $\mathrm{U}$ & U & U & $\mathrm{L}$ \\
\hline Castellanos,1997 & $\mathrm{U}$ & U & $\mathrm{U}$ & $\mathrm{U}$ & U & $\mathrm{U}$ & L \\
\hline Kurlan,2002 & $\mathrm{L}$ & $\mathrm{L}$ & $\mathrm{L}$ & U & $\mathrm{L}$ & U & $\mathrm{L}$ \\
\hline Silver, 2001 & $\mathrm{U}$ & $\mathrm{U}$ & $\mathrm{U}$ & U & $\mathrm{U}$ & $\mathrm{U}$ & $\mathrm{L}$ \\
\hline Gilbert, 2003 & $\mathrm{U}$ & U & $\mathrm{L}$ & $\mathrm{U}$ & $\mathrm{H}$ & $\mathrm{U}$ & $\begin{array}{c}\mathrm{U} \\
\text { (Continued) }\end{array}$ \\
\hline
\end{tabular}




\begin{tabular}{lccccccc}
\hline Study ID & $\begin{array}{c}\text { Random } \\
\text { sequence } \\
\text { generation }\end{array}$ & $\begin{array}{c}\text { Allocation } \\
\text { concealment }\end{array}$ & $\begin{array}{c}\text { Blinding of } \\
\text { participants }\end{array}$ & $\begin{array}{c}\text { Blinding } \\
\text { of outcome } \\
\text { assessment }\end{array}$ & $\begin{array}{c}\text { Incomplete } \\
\text { outcome } \\
\text { data }\end{array}$ & $\begin{array}{c}\text { Selective } \\
\text { reporting }\end{array}$ & $\begin{array}{c}\text { Other } \\
\text { sources of } \\
\text { bias }\end{array}$ \\
\hline Gilbert, 2000 & $\mathrm{L}$ & $\mathrm{L}$ & $\mathrm{L}$ & $\mathrm{U}$ & $\mathrm{L}$ & $\mathrm{U}$ & $\mathrm{L}$ \\
Kurlan,2012 & $\mathrm{U}$ & $\mathrm{U}$ & $\mathrm{U}$ & $\mathrm{U}$ & $\mathrm{H}$ & $\mathrm{U}$ & $\mathrm{L}$ \\
Goetz,1994 & $\mathrm{U}$ & $\mathrm{U}$ & $\mathrm{U}$ & $\mathrm{L}$ & $\mathrm{U}$ & $\mathrm{U}$ & $\mathrm{U}$ \\
Singer, 2001 & $\mathrm{L}$ & $\mathrm{U}$ & $\mathrm{L}$ & $\mathrm{U}$ & $\mathrm{U}$ & $\mathrm{U}$ & $\mathrm{H}$ \\
Lemmon,2015 & $\mathrm{L}$ & $\mathrm{U}$ & $\mathrm{L}$ & $\mathrm{U}$ & $\mathrm{U}$ & $\mathrm{U}$ & $\mathrm{U}$ \\
Bloch,2016 & $\mathrm{U}$ & $\mathrm{L}$ & $\mathrm{L}$ & $\mathrm{L}$ & $\mathrm{U}$ & $\mathrm{U}$ & $\mathrm{U}$ \\
Allen,2005 & $\mathrm{L}$ & $\mathrm{U}$ & $\mathrm{U}$ & $\mathrm{U}$ & $\mathrm{L}$ & $\mathrm{U}$ & $\mathrm{L}$ \\
Spencer,2008 & $\mathrm{U}$ & $\mathrm{U}$ & $\mathrm{U}$ & $\mathrm{U}$ & $\mathrm{H}$ & $\mathrm{U}$ & $\mathrm{L}$ \\
Silver,2001 & $\mathrm{L}$ & $\mathrm{L}$ & $\mathrm{L}$ & $\mathrm{U}$ & $\mathrm{H}$ & $\mathrm{U}$ & $\mathrm{L}$ \\
Mcconville,1992 & $\mathrm{U}$ & $\mathrm{U}$ & $\mathrm{U}$ & $\mathrm{U}$ & $\mathrm{L}$ & $\mathrm{U}$ & $\mathrm{L}$ \\
Zhao,2010 & $\mathrm{U}$ & $\mathrm{L}$ & $\mathrm{L}$ & $\mathrm{L}$ & $\mathrm{L}$ & $\mathrm{L}$ & $\mathrm{U}$ \\
Zheng,2016 & $\mathrm{U}$ & $\mathrm{U}$ & $\mathrm{L}$ & $\mathrm{U}$ & $\mathrm{L}$ & $\mathrm{L}$ & $\mathrm{L}$ \\
Li, 2009 & $\mathrm{L}$ & $\mathrm{U}$ & $\mathrm{U}$ & $\mathrm{U}$ & $\mathrm{L}$ & $\mathrm{L}$ & $\mathrm{U}$ \\
Wu,2010 & $\mathrm{U}$ & $\mathrm{U}$ & $\mathrm{U}$ & $\mathrm{U}$ & $\mathrm{U}$ & $\mathrm{U}$ & $\mathrm{U}$ \\
Wu,2009 & $\mathrm{L}$ & $\mathrm{U}$ & $\mathrm{U}$ & $\mathrm{U}$ & $\mathrm{U}$ & $\mathrm{L}$ & $\mathrm{U}$ \\
Toran,2005 & $\mathrm{U}$ & $\mathrm{U}$ & $\mathrm{U}$ & $\mathrm{U}$ & $\mathrm{U}$ & $\mathrm{U}$ & $\mathrm{H}$ \\
Nicolson,2005 & $\mathrm{U}$ & $\mathrm{U}$ & $\mathrm{U}$ & $\mathrm{U}$ & $\mathrm{L}$ & $\mathrm{U}$ & $\mathrm{L}$ \\
\hline
\end{tabular}

Note: H: high risk; L:low risk; U:unclear

\section{Selective norepinephrine reuptake inhibitor}

Atomoxetine from two trials was superior to placebo in children (YGTSS: WMD $=-2.767,95 \%$ CI, -4.649 -0.882 ; CGI: $\mathrm{WMD}=-0.644,95 \% \mathrm{CI},-0.910--0.378$ ) (Figure 4 and 5).

\section{Smoking cessation agent}

For nicotine patch, the pooled result of YGTSS showed a positive effect (WMD $=-7.018,95 \% \mathrm{CI},-8.252$ -5.783) (Figure 6). It's worth noting that Silver et al. reported a favorable effect of nicotine patch in children (YGTSS: $\mathrm{WMD}=-7.100,95 \% \mathrm{CI},-8.342-\quad-5.858$ ), however, another trial by McConville et al. including patients with the mean age of 22 discovered that nicotine patch might not improve tics (YGTSS: WMD $=-0.300$, 95\% CI, -11.511- 10.911). The explanation to these might be that the two studies included subjects with different age range. Moreover, McConville et al. also evaluated the effect of nicotine patch plus haloperidol compared to placebo and found that there was no significant difference between treatments in the scale of YGTSS (WMD $=-6.400$, 95\% CI, -16.549- 3.749) and CGI (WMD $=-0.800,95 \% \mathrm{CI}$, -1.901- 0.301).

\section{Traditional Chinese medicine}

Traditional Chinese Medicine (TCM) (NingDong Granule and 5-Ling Granule) all showed positive effects in the scale of YGTSS (NingDong Granule: WMD $=-7.100$, $95 \%$ CI, -10.430- -3.770; 5-Ling Granule: $\mathrm{WMD}=-11.300$, 95\% CI, -14.208- -8.392).

\section{HT3-receptor antagonists}

The study of ondansetron was conducted by Toran et al. in the mixed patients (mean age was 21.7 \pm 9.14 ) and failed to show a positive effect (YGTSS: WMD $=-2.000$, 95\% CI, -9.203- 5.203; TSGS: WMD $=-2.680,95 \%$ CI, -16.742-11.382). Conversely, metoclopramide tested in a children trial was superior to placebo (YGTSS: WMD=$5.900,95 \%$ CI, $-10.147--1.653$, CGI: WMD $=-1.000,95 \%$ CI, -1.639- -0.361).

\section{Head-to-head comparisons}

Table 8 to Table 10 demonstrated the efficacy of pharmacotherapies compared to each other in each outcome measure.

Three interventions (from 11 studies) out of 16 interventions including NingDong Granule plus 
Table 4: Effect sizes of pharmacological interventions compared with placebo using the outcome scale of YGTSS for patients with Tourette syndrome

\begin{tabular}{llcc}
\hline Concomitant Drug/Study & \multicolumn{3}{c}{ YGTSS } \\
\hline Author (Year) & $\mathrm{N}^{1}$ & Age $^{2}$ & WMD \\
\hline
\end{tabular}

Alpha-2 adrenergic agonist agent

Guanfacine

Scahill,2001

Cummings,2002

Anticonvulsant

Levetiracetam

Smith-hicks, 2007

Topiramate

Jankovic, 2010

Antidepressant

Desipramine

$$
\text { Spencer,2002 }
$$

1

Fluoxetine

$$
\text { Scahill,1997 }
$$

Antipsychotic agent

Atypical Antipsychotic agent

Ziprasidone

Sallee, 1999

Risperidone

Scahill,2003

1

1

1

1

1

b

$-9.290(-16.697,-1.883)$

$-4.596(-8.798,-0.393)$

$-4.500(-8.939,-0.061)$

$-5.420(-18.461,7.621)$

a

$0.050(-16.175,16.275)$

a

$-16.000(-27.130,-4.870)$

4

1

a

$-6.900(-11.234,-2.566)$

$-6.400(-11.059,-1.741)$

$-7.100(-12.276,-1.924)$

Aripiprazole

Yoo,2013

1

a

$-5.100(-9.178,-1.022)$

Tiapride

Zheng,2016

1

a

$-11.700(-15.101,-8.299)$

Cannabis

Delta-9-tetrahydrocannabinol

Muller-vahl,2002

1

b

$-6.500(-19.174,6.174)$

CNS stimulant

Methylphenidate

7

$0.1 \mathrm{mg} / \mathrm{kg}$

Gadow, 1995

Gadow,2007

$0.3 \mathrm{mg} / \mathrm{kg}$

Gadow,1995

Gadow,2007
1

a

$0.035(-4.442,4.512)$

$-0.759(-9.270,7.752)$

1.000(-14.637,16.637)

$-1.500(-11.645,8.645)$

1.263(-7.307,9.832)

3.600(-12.907,20.107)

$0.400(-9.627,10.427)$ 


\section{Concomitant Drug/Study}

Author (Year)

$0.5 \mathrm{mg} / \mathrm{kg}$

Gadow,1995

Gadow,2007

Mixed dosage $(0.1,0.3,0.5 \mathrm{mg} / \mathrm{kg})$

Gadow,1999

Dopaminergic agent

Pergolide

Gilbert,2003

Gilbert,2000

Pramipexole

Kurlan,2012

Glutamate agonist

D-serine

Lemmon, 2015

N-Acetylcysteine

Bloch,2016

Glutamate antagonist

Riluzole

Lemmon,2015

Selective norepinephrine reuptake inhibitor

Atomoxetine

Allen,2005

Spencer,2008

Smoking cessation agent

Nicotine patch

Mcconville, 1992

Silver, 2001

Smoking cessation agent plus Antipsychotic drugs

Nicotine patch plus haloperidol

Mcconville, 1992

Traditional Chinese medicine

NingDong Granule

Zhao,2010

5-Ling Granule

Zheng,2016

\section{YGTSS}

$\begin{array}{cr}\mathbf{N}^{1} & \mathbf{A g} \\ & \\ 1 & \mathrm{a} \\ 1 & \mathrm{a} \\ & \mathrm{a} \\ 1 & \mathrm{a} \\ & \mathrm{a} \\ 2 & \mathrm{a} \\ 1 & \\ 1 & \end{array}$

$-0.706(-9.119,7.707)$

$0.800(-15.021,16.621)$

$-1.300(-11.234,8.634)$

$0.600(-10.347,11.547)$

$-13.167(-20.553,-5.781)$

$-8.800(-18.761,1.161)$

$-18.500(-29.508,-7.492)$

$-0.150(-2.277,1.977)$

a

$-2.600(-19.985,14.785)$

$2.200(-2.830,7.230)$

a

$-4.100(-23.452,15.252)$

$-2.767(-4.649,-0.882)$

$-2.500(-5.023,0.023)$

$-3.100(-5.931,-0.269)$

$-7.018(-8.252,-5.783)$

$-0.300(-11.511,10.911)$

$-7.100(-8.342,-5.858)$

a

$-6.400(-16.549,3.749)$

b

$-7.100(-10.430,-3.770)$

$-11.300(-14.208,-8.392)$

(Continued) 


\begin{tabular}{|c|c|c|c|}
\hline \multirow{2}{*}{$\begin{array}{l}\text { Concomitant Drug/Study } \\
\text { Author (Year) }\end{array}$} & \multicolumn{3}{|c|}{ YGTSS } \\
\hline & $\mathbf{N}^{1}$ & Age $^{2}$ & WMD \\
\hline \multicolumn{4}{|l|}{ 5HT3-receptor antagonists } \\
\hline \multicolumn{4}{|l|}{ Ondansetron } \\
\hline Toran,2005 & 1 & $\mathrm{~b}$ & $-2.000(-9.203,5.203)$ \\
\hline \multicolumn{4}{|l|}{ Metoclopramide } \\
\hline Nicolson,2005 & 1 & $\mathrm{a}$ & $-5.900(-10.147,-1.653)$ \\
\hline
\end{tabular}

Note: Significant results are in bold.

1: N:number of studies;

2: a represented that the subjects included in the study were children; $b$ represented that the subjects included in the study

were mixed participation (children and adult).

haloperidol, Qufeng Zhidong Recipe, and olanzapine showed significant positive effects compared to another interventions including haloperidol, haloperidol plus trihexyphenidyl, and pimozide, respectively. It's worth noting that among the above comparisons only one (Qufeng Zhidong Recipe versus haloperidol plus trihexyphenidyl) incorporated two trials, while the rest merely incorporated one.

Four studies tested the efficacy of TCM alone or plus western medicines compared to western medicines. One trial evaluated the comparison of NingDong Granule plus haloperidol versus haloperidol. Two trials evaluated the comparison of Qufeng Zhidong Recipe versus haloperidol plus trihexyphenidyl. The above two comparisons all yielded significant differences in the measure of YGTSS (WMD $=-4.260,95 \% \mathrm{CI},-7.284--1.236$; $\mathrm{WMD}=-16.886$, 95\% CI, -18.073- -15.700 (Figure 7); respectively). Another study was about a larger multisite, double-blind randomized, placebo-controlled trial of 603 children randomized to 5-Ling Granule ( $\mathrm{N}=363)$, tiapride $(\mathrm{N}=123)$ or placebo $(\mathrm{N}=117)$ for 8 weeks. This trial failed to indicate a significant positive effect of 5-Ling Granule compared to tiapride (WMD $=0.400,95 \% \mathrm{CI},-2.304$ 3.104).

Three researches tested the different efficacy among diverse types of antipsychotic agents. Only olanzapine was found to be less effective than pimozide in the outcome of TSGS WMD=-13.000, 95\% CI,-15.504- -10.496). Other comparisons, including aripiprazole versus risperidone and risperidone versus pimozide, yielded no significant differences.

Studies indicated that clonidine was not significant different from levetiracetam (YGTSS: WMD $=-2.000,95 \%$ CI,-15.455- 11.455, CGI: WMD=-0.100, 95\% CI, -0.6750.475 ) and risperidone (YGTSS: $\mathrm{WMD}=-2.900,95 \%$ CI, -15.142- 9.342). Similarly, there were no significant differences between fluvoxamine versus sulpiride (YGTSS: WMD $=5.000,95 \% \mathrm{CI},-19.459-29.459$ ) and D-serine versus riluzole (YGTSS: WMD $=1.500,95 \% \mathrm{CI}$, -16.106- 19.106).

\section{Adverse effect}

Detailed adverse effects of pharmacological interventions were displayed in Supplementary Table (1-14).

Of note, antipsychotic agents, especially typical neuroleptics, were associated with severer adverse effects, including weight gain, akathisia, and acute dystonia, than alpha-2 adrenergic agonist agents. Weight gain was a concern for the typical neuroleptics.

\section{Sensitivity analyses and publication bias}

None of the interventions included more than two studies in meta-analysis, so sensitivity analyses and publication bias were not assessed according to the recommendations of the Cochrane Handbook for Systematic Reviews of Interventions (www. cochranehandbook.org).

\section{DISCUSSION}

Meta-analysis suggested that alpha-2 adrenergic agonist agents and atypical antipsychotic agents were effective in improving tics, which included the maximum number of trials. Although typical antipsychotic agents were widely used in patients suffered from tics, certain evidences were scarce. Besides, according to head-tohead trials, there were no significant differences among the comparisons of antipsychotic agents and alpha-2 adrenergic agonist agents in efficacy, but the side effects among them were diverse. Antipsychotics agents, especially typical antipsychotic agents, were associated with severer side-effects than alpha-2 adrenergic agonist agents. Similar to several clinical guidelines [10, 23], typical and atypical neuroleptics were recommended with several evidences, but side effects restricted their use as first-line options.

In addition, for agents (e.g. CNS stimulants and atomoxetine) primarily used to manage comorbid tics and 
Table 5: Effect sizes of pharmacological interventions compared with placebo using the outcome scale of CGI for patients with Tourette syndrome

\begin{tabular}{|c|c|c|c|}
\hline \multirow{2}{*}{$\begin{array}{l}\text { Concomitant Drug/Study (N) } \\
\text { Author (Year) } \\
\end{array}$} & \multicolumn{3}{|c|}{ CGI } \\
\hline & $\mathbf{N}^{1}$ & Age $^{2}$ & WMD \\
\hline \multicolumn{4}{|l|}{ Alpha-2 adrenergic agonist agent } \\
\hline \multicolumn{4}{|l|}{ Clonidine } \\
\hline Leckman,1991 & 1 & $\mathrm{~b}$ & $-0.600(-0.996,-0.204)$ \\
\hline \multicolumn{4}{|l|}{ Anticonvulsant } \\
\hline \multicolumn{4}{|l|}{ Levetiracetam } \\
\hline Smith-hicks,2007 & 1 & a & $0.090(-0.572,0.752)$ \\
\hline \multicolumn{4}{|l|}{ Antipsychotic agent } \\
\hline \multicolumn{4}{|l|}{ Atypical Antipsychotic agent } \\
\hline \multicolumn{4}{|l|}{ Ziprasidone } \\
\hline Sallee, 1999 & 1 & $\mathrm{a}$ & $-0.700(-1.407,0.007)$ \\
\hline \multicolumn{4}{|l|}{ Risperidone } \\
\hline Dion, 2002 & 1 & $\mathrm{~b}$ & $-0.650(-1.207,-0.093)$ \\
\hline \multicolumn{4}{|l|}{ CNS stimulant } \\
\hline \multicolumn{4}{|l|}{ Methylphenidate } \\
\hline \multicolumn{4}{|c|}{ Mixed dosage $(0.1,0.3,0.5 \mathrm{mg} / \mathrm{kg})$} \\
\hline Gadow,1999 & 1 & a & $0.000(-0.410,0.410)$ \\
\hline \multicolumn{4}{|c|}{ Gamma-aminobutyric acid-B receptor agonist } \\
\hline \multicolumn{4}{|l|}{ Baclofen } \\
\hline Singer,2001 & 1 & $\mathrm{a}$ & $-0.900(-1.497,-0.303)$ \\
\hline \multicolumn{4}{|l|}{ Glutamate agonist } \\
\hline \multicolumn{4}{|l|}{ N-Acetylcysteine } \\
\hline Bloch,2016 & 1 & a & $0.100(-0.622,0.822)$ \\
\hline \multicolumn{4}{|c|}{ Selective norepinephrine reuptake inhibitor } \\
\hline Atomoxetine & 2 & a & $-0.644(-0.910,-0.378)$ \\
\hline Allen, 2005 & 1 & a & $-0.600(-0.957,-0.243)$ \\
\hline Spencer,2008 & 1 & a & $-0.700(-1.099,-0.301)$ \\
\hline \multicolumn{4}{|c|}{ Smoking cessation agent plus Antipsychotic drugs } \\
\hline \multicolumn{4}{|c|}{ Nicotine patch plus haloperidol } \\
\hline Mcconville, 1992 & 1 & $\mathrm{~b}$ & $-0.800(-1.901,0.301)$ \\
\hline \multicolumn{4}{|l|}{ 5HT3-receptor antagonists } \\
\hline \multicolumn{4}{|l|}{ Metoclopramide } \\
\hline Nicolson,2005 & 1 & a & $-1.000(-1.639,-0.361)$ \\
\hline
\end{tabular}

Note: Significant results are in bold.

1: N:number of studies;

2: a represented that the subjects included in the study were children; b represented that the subjects included in the study were mixed participation (children and adult). 
Table 6: Effect sizes of pharmacological interventions compared with placebo using the outcome scale of TSGS for patients with Tourette syndrome

\begin{tabular}{|c|c|c|c|}
\hline \multirow{2}{*}{$\begin{array}{l}\text { Concomitant Drug/Study (N) } \\
\text { Author (Year) }\end{array}$} & \multicolumn{3}{|c|}{ TSGS } \\
\hline & $\mathbf{N}^{1}$ & Age $^{2}$ & WMD \\
\hline \multicolumn{4}{|l|}{ Analgesic } \\
\hline \multicolumn{4}{|l|}{ Naltrexone } \\
\hline Kurlan,1991 & 1 & $\mathrm{~b}$ & $-0.100(-6.426,6.226)$ \\
\hline \multicolumn{4}{|l|}{ Propoxyphene } \\
\hline Kurlan,1991 & 1 & $\mathrm{~b}$ & $-8.700(-14.711,-2.689)$ \\
\hline \multicolumn{4}{|l|}{ Antipsychotic agent } \\
\hline \multicolumn{4}{|l|}{ Typical Antipsychotic agent } \\
\hline \multicolumn{4}{|l|}{ Haloperidol } \\
\hline Sallee, 1997 & 1 & $\mathrm{a}$ & $-6.100(-15.361,3.161)$ \\
\hline \multicolumn{4}{|l|}{ Pimozide } \\
\hline Sallee, 1997 & 1 & a & $-9.700(-18.436,-0.964)$ \\
\hline \multicolumn{4}{|l|}{ Cannabis } \\
\hline \multicolumn{4}{|l|}{ Delta-9-tetrahydrocannabinol } \\
\hline Muller-vahl,2002 & 1 & $\mathrm{~b}$ & $-6.500(-15.652,2.652)$ \\
\hline \multicolumn{4}{|l|}{ 5HT3-receptor antagonists } \\
\hline \multicolumn{4}{|l|}{ Ondansetron } \\
\hline Toran,2005 & 1 & $\mathrm{~b}$ & $-2.680(-16.742,11.382)$ \\
\hline
\end{tabular}

Note: Significant results are in bold.

1: N:number of studies;

2: a represented that the subjects included in the study were children; b represented that the subjects included in the study were mixed participation (children and adult).

Table 7: Effect sizes of pharmacological interventions compared with placebo using the outcome scale of TSSS for patients with Tourette syndrome

\begin{tabular}{|c|c|c|c|}
\hline Concomitant Drug/Study (N) & & TSSS & \\
\hline Author (Year) & N1 & Age2 & WMD \\
\hline \multicolumn{4}{|l|}{ Antipsychotic agent } \\
\hline \multicolumn{4}{|l|}{ Typical Antipsychotic agent } \\
\hline \multicolumn{4}{|l|}{ Haloperidol } \\
\hline Shapiro, 1989 & 1 & $\mathrm{~b}$ & $-1.700(-3.006,-0.394)$ \\
\hline \multicolumn{4}{|l|}{ Pimozide } \\
\hline Shapiro, 1989 & 1 & $\mathrm{~b}$ & $-0.400(-1.952,1.152)$ \\
\hline \multicolumn{4}{|l|}{ Atypical Antipsychotic agent } \\
\hline \multicolumn{4}{|l|}{ Risperidone } \\
\hline Dion,2002 & 1 & $\mathrm{~b}$ & $-1.070(-2.092,-0.048)$ \\
\hline
\end{tabular}

Note: Significant results are in bold.

1: N:number of studies;

2: a represented that the subjects included in the study were children; b represented that the subjects included in the study were mixed participation (children and adult). 
ADHD, evidences suggested that CNS stimulants might not exacerbate tics and atomoxetine might improve tics. For agents (e.g. fluoxetine) primarily used to manage comorbid tics and obsessive compulsive disorder/ obsessive compulsive symptom, evidences suggested that fluoxetine might not exacerbate tics. These results were in close agreement with relevant systematic reviews and meta-analyses which suggested that CNS stimulants might not deteriorate tics $[24,80]$. Nonetheless, clinical reports found that tics might become worse in patients undergoing high doses of stimulants [59].

In this meta-analysis, another significant discovery for clinical practice was the positive efficacy of TCM. Both of NingDong Granule and 5-Ling Granule might be more efficacious than placebo. NingDong Granule plus haloperidol might be more efficacious than haloperidol and Qufeng Zhidong Recipe might be more efficacious than haloperidol plus trihexyphenidyl. Unlike western medications, these three TCM (NingDong Granule, 5-Ling Granule, and Qufeng Zhidong Recipe) were specifically developed for tics. Traditional Chinese medicine believed that tics belonged to the sort of chronic infantile convulsion and hyperspasmia, and the major cause was yin-insufficiency in heart and liver [73, 81]. NingDong Granule, composed of eight different Chinese herbs and natural materials, could relieve convulsion and spasm by nourishing heart and liver yin [82]. Preclinical trials found that NingDong Granule improved the stereotypical behaviour of apomorphine-induced tics rats, an animal model of tics, by suppressing the dopamine system [28, 83]. 5-Ling Granule, a patented polyherbal product manufactured from 11 herbal materials, suppressed hyperactivity and tranquilize fidgetiness [74, 84]. Preclinical trials found that 5-Ling Granule suppressed head twitching and stereotyped movement in rat model induced by 3, 3'-iminodipropionitrile (IDPN), a synthetic neurotoxin. It also suppressed the stimulant amphetamine (AMP)-induced hyperactivity and irritability in mice. It's worth noting that different from haloperidol, a typical antipsychotic agent, 5-Ling Granule did not changed CNS excitability or spatial cognition [84]. Western medications for the management of tics were initially designed for other diseases, and accompanied by many adverse effects. For example, antipsychotic agents cut off dopamine receptors (act as dopamine antagonists), which generated the risk of extrapyramidal symptoms (EPS), dystonia, parkinsonism, and tardive dyskinesia. Traditional Chinese medicine also believed that the basic pathogenesis of tics was generally internal Lung-wind upsetting. Qufeng Zhidong Recipe was formulated by modifying the classic recipe for dispelling external wing [77].

However, firm conclusions were unable to draw due to the relatively tiny number of trials and findings needed to be replicated in more trials.

In general, the findings aligned with the review conducted by Craig Whittington et al [85]. Results showed that antipsychotic agents and alpha-2 adrenergic agonist agents demonstrated positive compared with placebo in improving tics. Different from their work which only included placebo-controlled trials, this review included head-to-head trials as well. Besides, this review included more comprehensive kinds of pharmacotherapies, such as TCM (NingDong Granule and 5-Ling Granule) and glutamate modulators (D-serine, N-Acetylcysteine and riluzole). The efficacy of different doses of methylphenidate were evaluated as well.

Furthermore, this results largely agreed with previous review by Chris Hollis et al.[86], despite this meta-analysis using various outcome measures. However, they only included medication with marketing authorisation in North America, Europe or Australasia, their results of pharmacotherapies were not comprehensive. Besides, they conducted a survey to capture qualitative and descriptive data on young people's experiences of treatment.

Concerning the review by Weisman et al. [87], it was too mechanistic to standardize the information of existing studies on pharmacotherapies for tics to perform a meta-analysis. They concentrated solely on the efficacy of FDA-approved antipsychotic agent or alpha-2 adrenergic agonists for treating tics, which failed to evaluate the side-effect profile and other kinds of pharmacological treatments. In terms of statistical analysis, although they performed a subgroup analysis by classifying studies according to comorbid ADHD condition and metaregression to examine the relationship between efficacy and several natural variables, including trial duration, trial methodological quality, and percentage of subjects with TS, the number of studies included in each category was only five. The statistical power with such few studies was too low to detect meaningful results. So even though these factors possibly might influence the outcomes, relevant analyses were not conducted in this review.

A number of superiorities existed in this research. First, this review was reported based on PRISMA recommendations [88]. Second, in order to minimize error, three independent investigators were used in the part of literature screening, data extraction and risk of bias evaluation [89]. Third, in order to make a comprehensive assessment of pharmacological agents, all trials with subjects at any age suffering from tics were included in this systematic review. Fourth, this review not only included placebo controlled trials, but also assessed headto-head trials, which were more comprehensive. Fifth and finally, the results with clear age of subjects were displayed.

However, several limitations needed to be considered when comprehending this meta-analysis.

First, despite the above clear results, the available studies of each intervention were few in number, which lacked sufficient statistical power for realistically evaluating the efficacy and publication bias. There was 
only one research about many treatments. Especially for head-to-head comparisons, the conclusions of no significant difference might not mean no difference in practice, which might be caused by few evidences with insufficient statistical power. Besides, the methodological quality of several included studies were not very well. As a consequence, conclusions were not completely certain and required repetition in more amount of populations. Second, the included trials evaluated short-term efficacy and safety in general, which meant that long-term outcomes were uncertain. Especially for side-effects, the reporting in controlled trials were less than desirable and a number of trials even did not recorded. Given that uncontrolled longer-term trials applying agents to tics and other disorders could provide more full-scale estimation, the overall assessment of adverse effects should take these studies into consideration. But these were beyond the range of this review, which made more reliable and practical adverse effects to be under-estimated. However, some important adverse effects were still detected in this

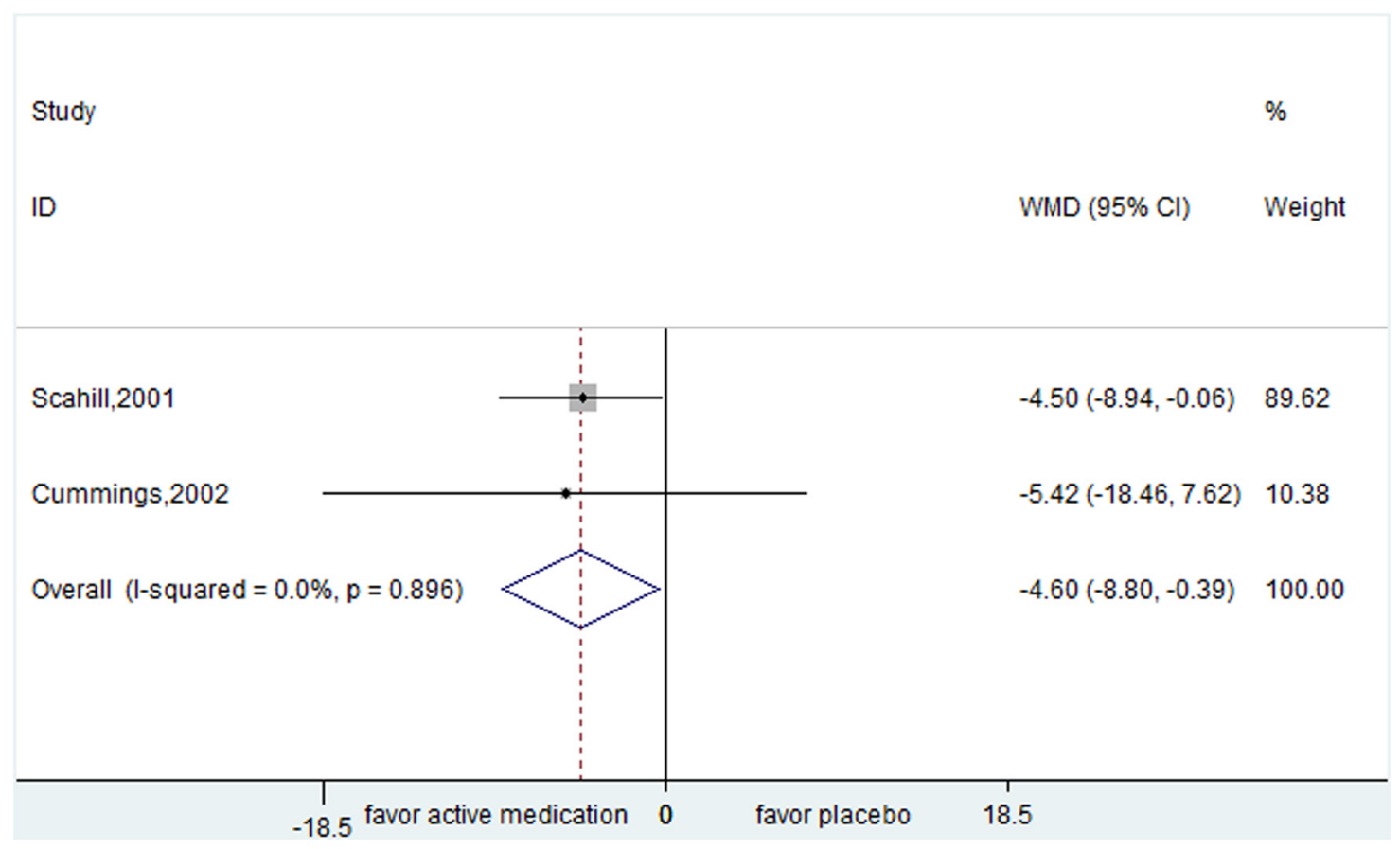

Figure 2: Efficacy of guanfacine compared with placebo for the treatment of tics in the outcome of Yale Global Tic Severity Scale.

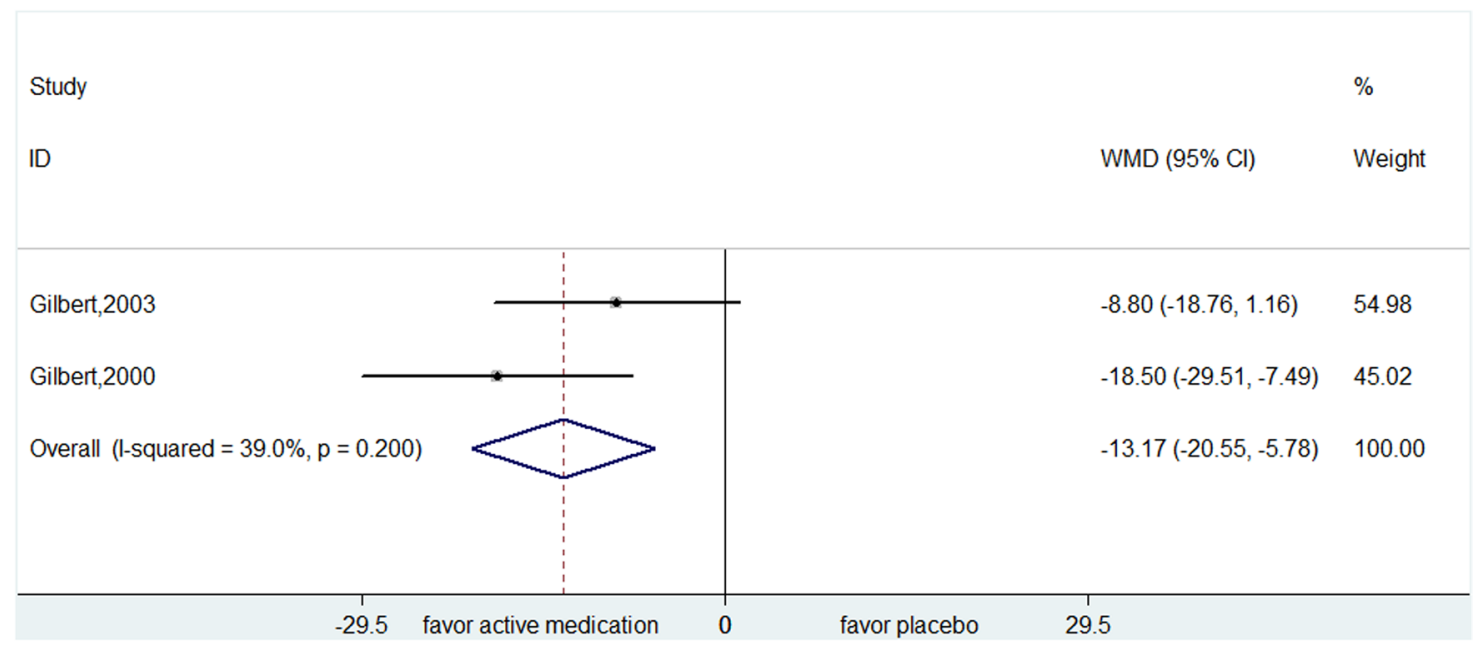

Figure 3: Efficacy of pergolide compared with placebo for the treatment of tics in the outcome of Yale Global Tic Severity Scale. 
review. By limiting to controlled trials, more dependable estimates of the percentage of subjects suffering from adverse events were able to be obtained. Third, although complications such as ADHD might impact the effect of alpha-2 agonists agents [87], the influence of complications on effect size was not measured, because the number of studies on each intervention was too small with inadequate statistical power to identify difference. What's more, meta-analytic approach is not the best tool to identify the influence of complications because the potential variability is usually correlated among trials. For instance, some subjects in trials with long-term

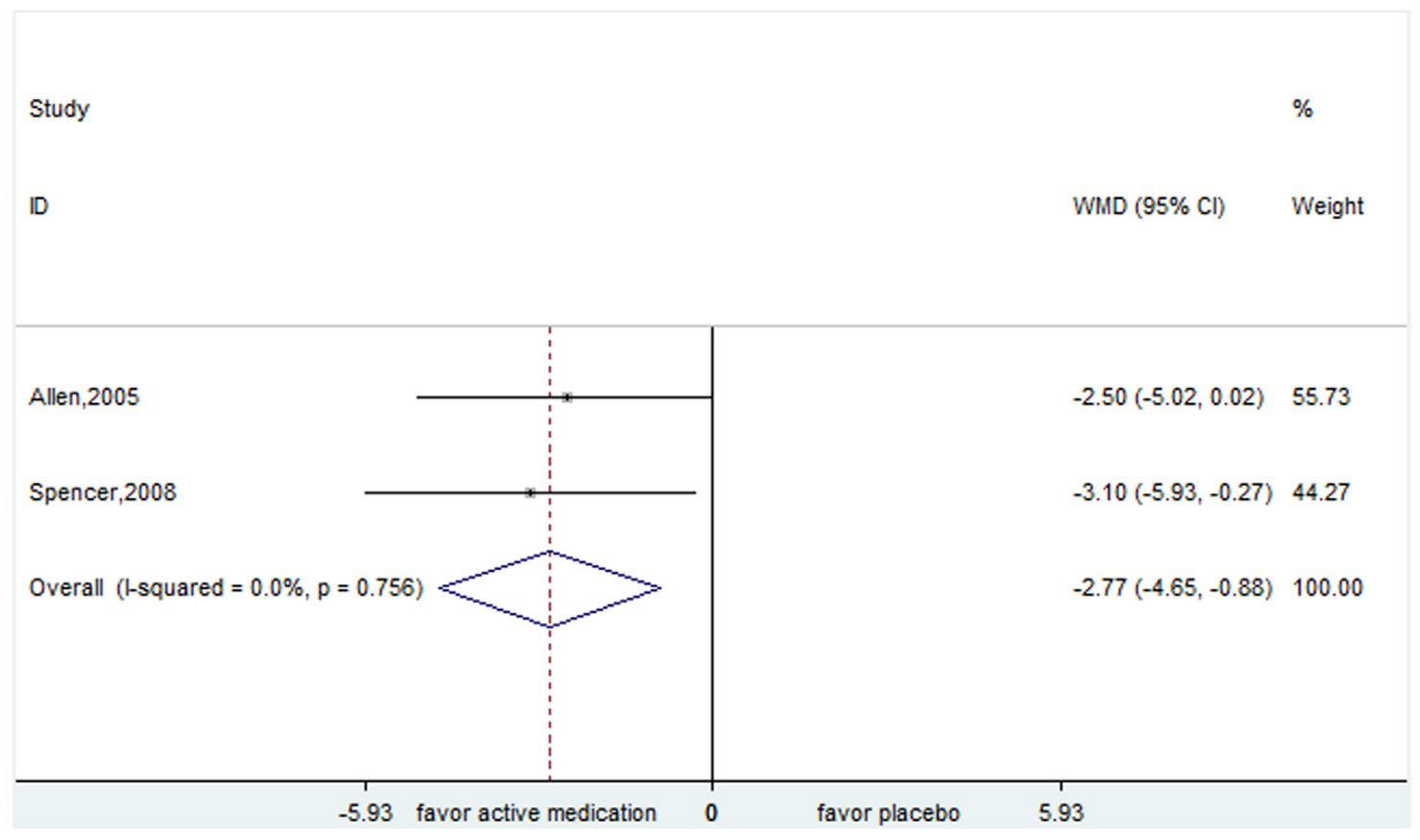

Figure 4: Efficacy of atomoxetine compared with placebo for the treatment of tics in the outcome of Yale Global Tic Severity Scale.

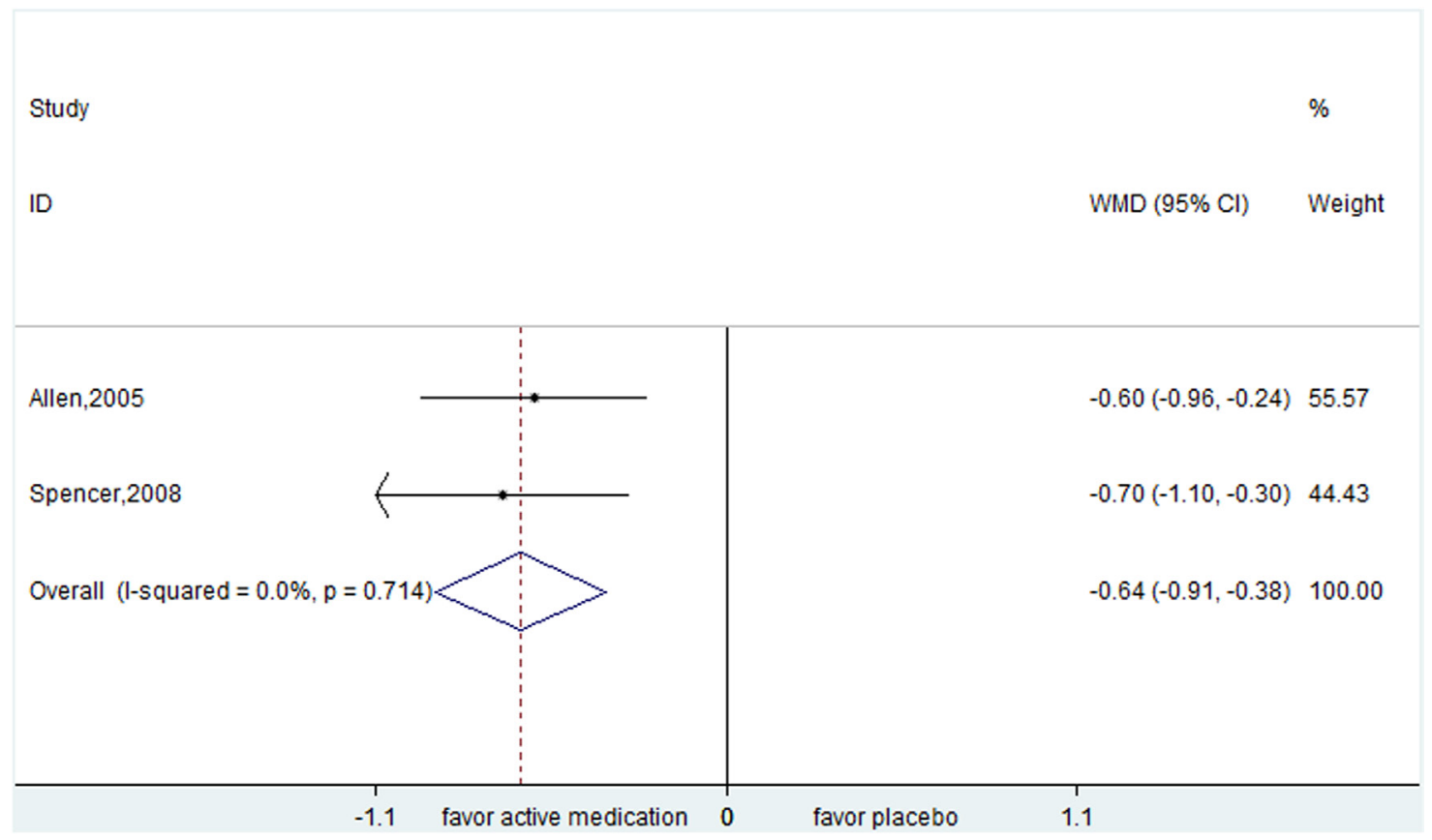

Figure 5: Efficacy of atomoxetine compared with placebo for the treatment of tics in the outcome of Clinical Global Impression Scale. 
duration tend to develop new complications in the period of research, which are very inconvenience to count in original trials and difficult to analyze in meta-analyses. Fourth, the effects of pharmacotherapies on motor and vocal tics separately were failed to be evaluated due to the inconsistent reporting among studies. Fifth, the outcome measures included in this review might be not very appropriate. For example, the quality of YGTSS to measure effects after 8 weeks is uncertainty, and CGI is a short inventory and not tic specific scale compared to the YGTSS. Last but not least, failing to identify unpublished trials might generated publication bias.

Future studies should include larger sample sizes to help minimize random error. Longer-term and head-to-

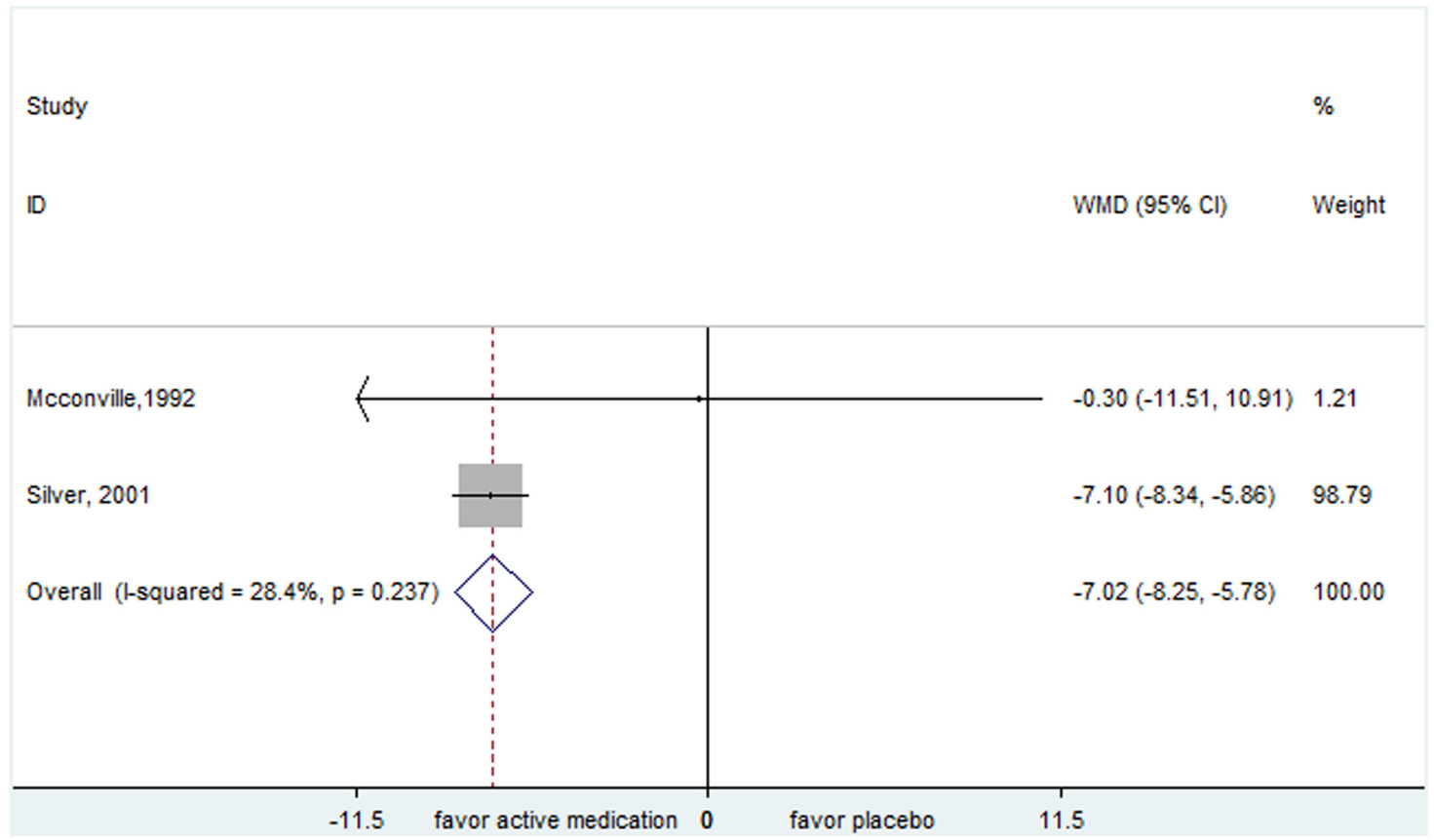

Figure 6: Efficacy of nicotine patch compared with placebo for the treatment of tics in the outcome of Yale Global Tic Severity Scale.

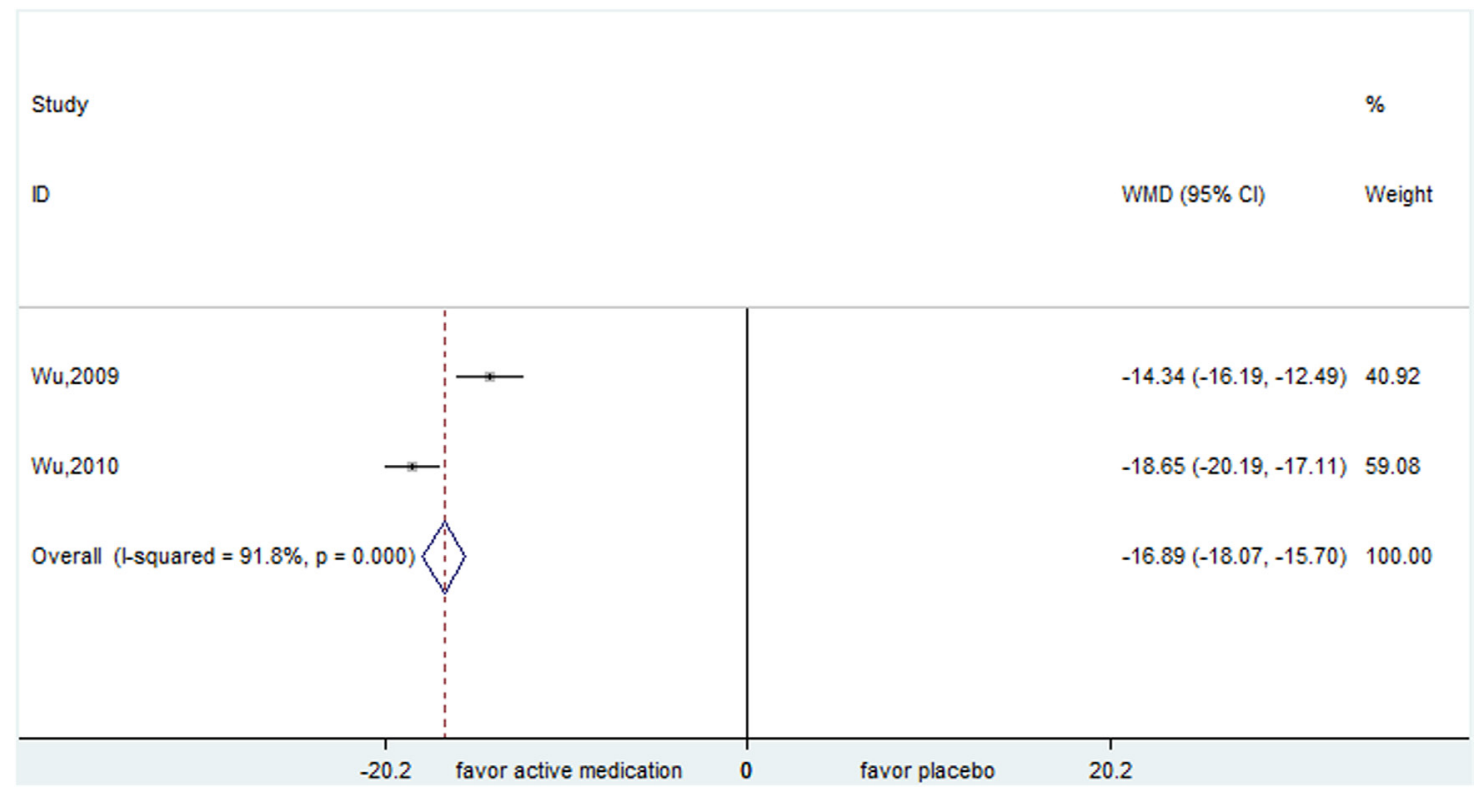

Figure 7: Efficacy of Qufeng Zhidong Recipe compared with Haloperidol plus trihexyphenidyl for the treatment of tics in the outcome of Yale Global Tic Severity Scale. 
Table 8: Effect sizes of pharmacological interventions from head-to head trials using the outcome scale of YGTSS for patients with Tourette syndrome

\begin{tabular}{|c|c|c|c|}
\hline \multirow{2}{*}{$\begin{array}{l}\text { Concomitant Drug/Study } \\
\text { Author (Year) }\end{array}$} & \multicolumn{3}{|c|}{ YGTSS } \\
\hline & $\mathbf{N}^{1}$ & $\operatorname{Age}^{2}$ & WMD \\
\hline \multicolumn{4}{|c|}{ NingDong Granule plus Haloperidol VS Haloperidol } \\
\hline Li,2009 & 1 & a & $-4.260(-7.284,-1.236)$ \\
\hline $\begin{array}{l}\text { Qufeng Zhidong Recipe VS Haloperidol plus } \\
\text { Trihexyphenidyl }\end{array}$ & 2 & a & $-16.886(-18.073,-15.700)$ \\
\hline $\mathrm{Wu}, 2009$ & 1 & & $-14.340(-16.194,-12.486)$ \\
\hline $\mathrm{Wu}, 2010$ & 1 & & $-18.650(-20.193,-17.107)$ \\
\hline \multicolumn{4}{|l|}{ 5-Ling Granule VS Tiapride } \\
\hline Zheng,2016 & 1 & $\mathrm{a}$ & $0.400(-2.304,3.104)$ \\
\hline \multicolumn{4}{|l|}{ Clonidine VS Levetiracetam } \\
\hline Hedderick, 2009 & 1 & $\mathrm{~b}$ & $-2.000(-15.455,11.455)$ \\
\hline \multicolumn{4}{|l|}{ Clonidine VS Risperidone } \\
\hline Gaffney,2002 & 1 & $\mathrm{a}$ & $-2.900(-15.142,9.342)$ \\
\hline \multicolumn{4}{|l|}{ Aripiprazole VS Risperidone } \\
\hline Ghanizadeh,2014 & 1 & a & $-5.300(-11.430,0.830)$ \\
\hline \multicolumn{4}{|l|}{ Risperidone VS Pimozide } \\
\hline Gilbert,2003 b & 1 & $\mathrm{a}$ & $-9.000(-23.949,5.949)$ \\
\hline \multicolumn{4}{|l|}{ Fluvoxamine VS Sulpiride } \\
\hline George, 1993 & 1 & $\mathrm{~b}$ & $5.000(-19.459,29.459)$ \\
\hline \multicolumn{4}{|l|}{ D-serine VS Riluzole } \\
\hline Yoo,2013 & 1 & $\mathrm{a}$ & $1.500(-16.106,19.106)$ \\
\hline
\end{tabular}

Note: Significant results are in bold.

1: N:number of studies;

2: a represented that the subjects included in the study were children; b represented that the subjects included in the study were mixed participation (children and adult).

Table 9: Effect sizes of pharmacological interventions from head-to head trials using the outcome scale of CGI for patients with Tourette syndrome

\begin{tabular}{|c|c|c|c|}
\hline \multirow{2}{*}{$\begin{array}{l}\text { Concomitant Drug/Study (N) } \\
\text { Author (Year) }\end{array}$} & \multicolumn{3}{|c|}{ CGI } \\
\hline & $\mathbf{N}^{1}$ & $\operatorname{Age}^{2}$ & WMD \\
\hline \multicolumn{4}{|l|}{ Clonidine VS Levetiracetam } \\
\hline Hedderick, 2009 & 1 & $\mathrm{~b}$ & $-0.100(-0.675,0.475)$ \\
\hline \multicolumn{4}{|l|}{ Risperidone VS Pimozide } \\
\hline Gilbert,2003 b & 1 & $\mathrm{a}$ & $-1.000(-2.076,0.076)$ \\
\hline
\end{tabular}

Note: Significant results are in bold.

1: N:number of studies;

2: a represented that the subjects included in the study were children; b represented that the subjects included in the study were mixed participation (children and adult). 
Table 10: Effect sizes of pharmacological interventions from head-to head trials using the outcome scale of TSGS for patients with Tourette syndrome

\begin{tabular}{lccc}
\hline Concomitant Drug/Study & & \multicolumn{2}{c}{ TSGS } \\
\hline Author (Year) & $\mathbf{N}^{1}$ & Age $^{2}$ & WMD \\
\hline Olanzapine VS Pimozide & & & \\
$\quad$ Onofrj, 2000 & 1 & $\mathrm{~b}$ & $\mathbf{- 1 3 . 0 0 0 ( - 1 5 . 5 0 4 , - 1 0 . 4 9 6 )}$ \\
\hline
\end{tabular}

Note: Significant results are in bold.

1: N:number of studies;

2: a represented that the subjects included in the study were children; b represented that the subjects included in the study were mixed participation (children and adult).

head investigations will be required as well. What's more, about the data collection in original trial, recording some clinical confounders such as comorbidities would help comprehensively assess the efficacy of pharmacological strategies for patients suffering from tics.

\section{MATERIALS AND METHODS}

\section{Search strategy}

Our review was conducted and reported according to the PRISMA statement [90].

The literature retrieval was performed from 1976 to July 2015 (updated in Oct 2016) in Medline, Embase, The Cochrane Library, Chinese Biomedical Literature Database (CBM), China Knowledge Resource Integrated Database (CNKI), VIP Database, and Wanfang Database applying the following key words: tic or tics or tourette*. Citations of relevant studies were screened carefully for inclusion as well. Two reviewers independently screened the literatures referring to inclusion and exclusion criteria, and a third reviewer coordinated if they yielded disagreements.

\section{Inclusion and exclusion criteria}

Articles met the following requirements were included: 1) patients suffered from tics treating with pharmacotherapies; 2) the comparison group could be placebo or another pharmacotherapies; 3) trials should be randomized controlled trials (RCTs), crossover and parallel group controlled trials. In addition, only the latest report would be included if the trial has been reported many times. Discontinuation research would be excluded.

\section{Data extraction and quality assessment}

A pre-defined excel table was used to extract information about relevant characteristics of included studies such as participant, intervention, comparison, and outcome by two independent reviewers. Whether the trial was conducted in children or mixed participation (children and adult) was displayed as well. The primary efficacy outcome scale defined in this meta-analysis was the Yale Global Tic Severity Scale (YGTSS)[91], as it is the most frequently used scale with high acceptance, and the followings were defined as secondary outcomes: Clinical Global Impression Scale (CGI)[92], TS Global Scale (TSGS)[93], and TS Severity Scale (TSSS)[94]. In order to integrate the data extraction, some authors were contacted through emails for raw data. Anyone of the primary and secondary outcomes were extracted, if articles reported. Any disagreements were coordinated by a third reviewer to reach consensus. Risk of bias were evaluated based on methods recommended by Cochrane Handbook [95].

\section{Statistics}

Overall estimates of pooled weighted mean difference (WMD) with 95\% confidence interval (CI) were calculated for each outcome measure from baseline to endpoint other than standard mean difference (SMD), because WMD could reflect the original effect size change of each outcome measure and it's easier to understand clinically. Heterogeneities among trials were evaluated by $\mathrm{Q}$ and $\mathrm{I}^{2}$ statistic. If the results were $\mathrm{P}<0.1$ and $\mathrm{I}^{2}>50 \%$, the existence of heterogeneity was predicted and random effects model was used to summarize WMD and 95\% CI (high heterogeneity defined as $\mathrm{I}^{2}>75 \%$ ). If not, fixed effects model was used. In addition, sensitivity analysis would be performed by removing individual trial to test the reliability of findings. If any of the outcome included 10 or more studies, publication bias would be examined by funnel plot and Egger's test according to the recommendation of Cochrane Handbook [95, 96].

The above analyses were conducted with the help of STATA 11.0 (Stata Corporation, College Station, TX).

\section{CONCLUSIONS}

In summary, there were effective pharmacological treatments for the management of patients with tics, especially atypical antipsychotic agents and alpha-2 adrenergic agonist agents. And alpha-2 adrenergic agonist 
agents were associated with the optimal weigh between efficacy and safety. However, the limited trials and sample sizes discounted these findings. Future better studies are necessary to ascertain them. In clinical practice, the choice of pharmacological treatments should balance overall advantage and disadvantage.

\section{Author contributions}

1. Study concept and design: Zuojie Zhang, Chunsong Yang, Lingli Zhang.

2. Acquisition of data: Zuojie Zhang, Chunsong Yang, Bo Liu, Jing Zeng.

3. Quality Assessment: Qiusha Yi, Bo Liu, Jing Zeng.

4. Analysis and interpretation of data: Zuojie Zhang, Chunsong Yang, Qiusha Yi, Dan Yu.

5. Drafting of the manuscript: Zuojie Zhang, Chunsong Yang.

6. Critical revision of the manuscript for important intellectual content: Zuojie Zhang, Lingli Zhang.

7. Statistical analysis: Chunsong Yang, Qiusha Yi, Jing Zeng, Dan Yu.

8. Study supervision: Lingli Zhang, Chunsong Yang.

\section{ACKNOWLEDGMENTS}

We thank Group of People with Highest Risk of Drug Exposure of International Network for the Rational Use of Drugs, China for providing support to coordinate circulation of the manuscript to all co-authors and collect comments from all co-authors.

\section{CONFLICTS OF INTEREST}

The authors declare no conflicts of interest.

\section{FUNDING}

This work was supported by the Natural Science Foundation of China: Evidence-based establishment of evaluation index system for pediatric rational drug use in China (grant number 81373381).

\section{REFERENCES}

1. Wolstenholme LC, Crowder MJ. Diagnostic and Statistical Manual of Mental Disorders (DSM-IV). American Psychiatric Association. 2000.

2. Stern JS, Burza S, Robertson MM. Gilles de la Tourette's syndrome and its impact in the UK. Postgrad Med J. 2005; 81:12-19.

3. Leckman JF, Zhang H, Vitale A, Lahnin F, Lynch K, Bondi C, Kim Y, Peterson BS. Course of tic severity in Tourette syndrome: the first two decades. Pediatrics. 1998; 102: 14-19.
4. Robertson MM, Eapen V, Cavanna AE. The international prevalence, epidemiology and clinical phenomenology of Tourette syndrome: a cross-cultural perspective. J Psychosom Res. 2009; 67:475-83.

5. Scharf JM, Miller LL, Mathews CA, Ben-Shlomo Y. Prevalence of Tourette syndrome and chronic tics in the population-based Avon longitudinal study of parents and children cohort. J Am Acad Child Adolesc Psychiatry. 2012; 51:192-201.e5.

6. Wanderer S, Roessner V, Freeman R, Bock N, Rothenberger A, Becker A. Relationship of obsessive compulsive disorder to age-related comorbidity in children and adolescents with Tourette syndrome. J Dev Behav Pediatr. 2012; 33:124-33.

7. Pierce A, Rickards HE. Atypical antipsychotics for Tourette's Syndrome. Cochrane Database Syst Rev. 2009: CD008151.

8. Murphy TK, Lewin AB, Storch EA, Stock S, and American Academy of Child and Adolescent Psychiatry (AACAP) Committee on Quality Issues (CQI). Practice parameter for the assessment and treatment of children and adolescents with tic disorders. J Am Acad Child Adolesc Psychiatry. 2013; 52:1341-59.

9. Roessner V, Becker A, Banaschewski T, Freeman RD, Rothenberger A, and Tourette Syndrome International Database Consortium. Developmental psychopathology of children and adolescents with Tourette syndro me-impact of ADHD. Eur Child Adolesc Psychiatry. 2007; 16:S24-35.

10. The Subspecialty Group of Parkinson's disease and movement disorders, Society of neurology branch, Chinese medical association. The diagnosis and treatment of tourette syndrome guide. Chin J Neurol. 2009; 42:635-638

11. Erenberg G, Cruse RP, Rothner AD. The natural history of Tourette syndrome: a follow-up study. Ann Neurol. 1987; 22:383-85.

12. Roessner V, Schoenefeld K, Buse J, Bender S, Ehrlich S, Münchau A. Pharmacological treatment of tic disorders and Tourette Syndrome. Neuropharmacology. 2013; 68:143-9.

13. Eddy CM, Rizzo R, Gulisano M, Agodi A, Barchitta M, Calì P, Robertson MM, Cavanna AE. Quality of life in young people with Tourette syndrome: a controlled study. J Neurol. 2011; 258:291-301.

14. Cooper C, Robertson MM, Livingston G. Psychological morbidity and caregiver burden in parents of children with Tourette's disorder and psychiatric comorbidity. J Am Acad Child Adolesc Psychiatry. 2003; 42:1370-75.

15. Robertson MM. The Gilles de la Tourette syndrome: the current status. Br J Psychiatry. 1989; 154:147-69.

16. Robertson MM. The Gilles de la Tourette syndrome: the current status. Arch Dis Child Educ Pract Ed. 2012; 97:166-75.

17. Piacentini J, Woods DW, Scahill L, Wilhelm S, Peterson AL, Chang S, Ginsburg GS, Deckersbach T, Dziura J, LeviPearl S, Walkup JT. Behavior therapy for children with Tourette disorder: a randomized controlled trial. JAMA. 2010; 303:1929-1937. 
18. O'Connor K, Lavoie M, Blanchet P, St-Pierre-Delorme MÈ. Evaluation of a cognitive psychophysiological model for management of tic disorders: an open trial. Br J Psychiatry. 2016; 209:76-83.

19. Steeves T, McKinlay BD, Gorman D, Billinghurst L, Day L, Carroll A, Dion Y, Doja A, Luscombe S, Sandor P, Pringsheim T. Canadian guidelines for the evidence-based treatment of tic disorders:Behavioural therapy, deep brain stimulation, and transcranial magnetic stimulation. Can J Psychiatry. 2012; 57:144-151.

20. Pringsheim T, Doja A, Gorman D, McKinlay D, Day L, Billinghurst L, Carroll A, Dion Y, Luscombe S, Steeves T, Sandor P. Canadian guidelines for the evidencebased treatment of tic disorders: pharmacotherapy. Can J Psychiatry. 2012; 57:133-43.

21. Verdellen C, van de Griendt J, Hartmann A, Murphy T, and ESSTS Guidelines Group. European clinical guidelines for Tourette syndrome and other tic disorders. Part III: behavioural and psychosocial interventions. Eur Child Adolesc Psychiatry. 2011; 20:197-207.

22. Shapiro AK, Shapiro E. Treatment of Gilles de la Tourette's Syndrome with haloperidol. Br J Psychiatry. 1968; 114:345-50.

23. Roessner V, Plessen KJ, Rothenberger A, Ludolph AG, Rizzo R, Skov L, Strand G, Stern JS, Termine C, Hoekstra PJ, and ESSTS Guidelines Group. European clinical guidelines for Tourette syndrome and other tic disorders. Part II: pharmacological treatment. Eur Child Adolesc Psychiatry. 2011; 20:173-96.

24. Pringsheim T, Steeves T. Pharmacological treatment for Attention Deficit Hyperactivity Disorder (ADHD) in children with comorbid tic disorders. Cochrane Database Syst Rev. 2011; 3:CD007990.

25. Waldon K, Hill J, Termine C, Balottin U, Cavanna AE. Trials of pharmacological interventions for Tourette syndrome: a systematic review. Behav Neurol. 2013; 26:265-73.

26. Scahill L, Chappell PB, Kim YS, Schultz RT, Katsovich L, Shepherd E, Arnsten AF, Cohen DJ, Leckman JF. A placebo-controlled study of guanfacine in the treatment of children with tic disorders and attention deficit hyperactivity disorder. Am J Psychiatry. 2001; 158:1067-74.

27. Cummings DD, Singer HS, Krieger M, Miller TL, Mahone EM. Neuropsychiatric effects of guanfacine in children with mild tourette syndrome: a pilot study. Clin Neuropharmacol. 2002; 25:325-332.

28. Smith-Hicks CL, Bridges DD, Paynter NP, Singer HS. A double blind randomized placebo control trial of levetiracetam in Tourette syndrome. Mov Disord. 2007; 22:1764-70.

29. Scahill L, Riddle MA, King RA, Hardin MT, Rasmusson A, Makuch RW, Leckman JF. Fluoxetine has no marked effect on tic symptoms in patients with Tourette's syndrome: a double-blind placebo-controlled study. J Child Adolesc Psychopharmacol. 1997; 7:75-85.

30. Cuenca J, Glazebrook C, Kendall T, Hedderly T, Heyman I, Jackson G, Murphy T, Rickards H, Robertson M, Stern J, Trayner P, Hollis C. Perceptions of treatment for tics among young people with Tourette syndrome and their parents: a mixed methods study. BMC Psychiatry. 2015; 15:46.

31. Goetz CG, Tanner CM, Wilson RS, Carroll VS, Como PG, Shannon KM. Clonidine and Gilles de la Tourette's syndrome: double-blind study using objective rating methods. Ann Neurol. 1987; 21:307-10.

32. Leckman JF, Hardin MT, Riddle MA, Stevenson J, Ort SI, Cohen DJ. Clonidine treatment of Gilles de la Tourette's syndrome. Arch Gen Psychiatry. 1991; 48:324-328.

33. Du YS, Li HF, Vance A, Zhong YQ, Jiao FY, Wang HM, Wang MJ, Su LY, Yu DL, Ma SW, Wu JB. Randomized double-blind multicentre placebo-controlled clinical trial of the clonidine adhesive patch for the treatment of tic disorders. Aust N Z J Psychiatry. 2008; 42:807-13.

34. Singer HS, Brown J, Quaskey S, Rosenberg LA, Mellits ED, Denckla MB. The treatment of attention-deficit hyperactivity disorder in Tourette's syndrome: a doubleblind placebo-controlled study with clonidine and desipramine. Pediatrics.1995; 95:74-81.

35. Zhong YQ, Zhou WZ, Hu WG. [Randomized double-blind controlled study on treatment of tic disorders in children with transcutaneous patch of clonidine]. [Article in Chinese]. Zhonghua Er Ke Za Zhi. 2007; 45:785-87.

36. Niederhofer H, Staffen W, Mair A. A placebo-controlled study of lofexidine in the treatment of children with tic disorders and attention deficit hyperactivity disorder. J Psychopharmacol. 2003; 17:113-119.

37. Hedderick EF, Morris CM, Singer HS. Double-blind, crossover study of clonidine and levetiracetam in Tourette syndrome. Pediatr Neurol. 2009; 40: 420-425.

38. Kurlan R, Majumdar L, Deeley C, Mudholkar GS, Plumb $\mathrm{S}$, Como PG. A controlled trial of propoxyphene and naltrexone in patients with Tourette's syndrome. Ann Neurol. 1991; 30:19-23.

39. Jankovic J, Jimenez-Shahed J, Brown LW. A randomised, double-blind, placebo-controlled study of topiramate in the treatment of Tourette syndrome. J Neurol Neurosurg Psychiatry. 2010; 81:70-73.

40. George MS, Trimble MR, Robertson MM. Fluvoxamine and sulpiride in comorbid obsessive-compulsive disorder and Gilles de la Tourette syndrome. Hum Psychopharmacol. 1993; 8:327-334.

41. Spencer T, Biederman J, Coffey B, Geller D, Crawford M, Bearman SK, Tarazi R, Faraone SV. A double-blind comparison of desipramine and placebo in children and adolescents with chronic tic disorder and comorbid attention-deficit/hyperactivity disorder. Arch Gen Psychiatry. 2002; 59:649-56.

42. Feigin A, Kurlan R, McDermott MP, Beach J, Dimitsopulos T, Brower CA, Chapieski L, Trinidad K, Como P, Jankovic 
J. A controlled trial of deprenyl in children with Tourette's syndrome and attention deficit hyperactivity disorder. Neurology. 1996; 46:965-968.

43. Shapiro E, Shapiro AK, Fulop G, Hubbard M, Mandeli J, Nordlie J, Phillips RA. Controlled study of haloperidol, pimozide, and placebo for the treatment of Gilles de la Tourette's syndrome. Arch Gen Psychiatry. 1989; 46:722-730.

44. Sallee FR, Nesbitt L, Jackson C, Sine L, Sethuraman G. Relative efficacy of haloperidol and pimozide in children and adolescents with Tourette's disorder. Am J Psychiatry. 1997; 154:1057-62.

45. Shapiro AK, Shapiro E. Controlled study of pimozide vs placebo in Tourette's syndrome. J Am Acad Child Psychiatry. 1984; 23:161-173.

46. Sallee FR, Kurlan R, Goetz CG, Singer H, Scahill L, Law G, Dittman VM, Chappell PB. Ziprasidone treatment of children and adolescents with Tourette's syndrome: a pilot study. J Am Acad Child Adolesc Psychiatry. 2000; 39:292-99.

47. Scahill L, Leckman JF, Schultz RT, Katsovich L, Peterson BS. A placebo-controlled trial of risperidone in Tourette syndrome. Neurology. 2003; 60:1130-1135.

48. Dion Y, Annable L, Sandor P, Chouinard G. Risperidone in the treatment of Tourette syndrome: a double-blind, placebo-controlled trial. J Clin Psychopharmacol. 2002; 22:31-39.

49. Gaffney GR, Perry PJ, Lund BC, Bever-Stille KA, Arndt S, Kuperman S. Risperidone versus clonidine in the treatment of children and adolescents with Tourette's syndrome. J Am Acad Child Adolesc Psychiatry. 2002; 41:330-336.

50. Gilbert DL, Batterson JR, Sethuraman G, Sallee FR. Tic reduction with risperidone versus pimozide in a randomized, double-blind, crossover trial. J Am Acad Child Adolesc Psychiatry. 2004; 43:206-214.

51. Yoo HK, Joung YS, Lee JS, Song DH, Lee YS, Kim JW, Kim BN, Cho SC. A multicenter, randomized, doubleblind, placebo-controlled study of aripiprazole in children and adolescents with Tourette's disorder. J Clin Psychiatry. 2013; 74:e772-80.

52. Ghanizadeh A, Haghighi A. Aripiprazole versus risperidone for treating children and adolescents with tic disorder: a randomized double blind clinical trial. Child Psychiatry Hum Dev. 2014; 45:596-603.

53. Eggers C, Rothenberger A, Berghaus U. Clinical and neurobiological findings in children suffering from tic disease following treatment with tiapride. Eu $\mathrm{r}$ Arch Psychiatry Neurol Sci. 1988; 237:223-29.

54. Onofrj M, Paci C, D'Andreamatteo G, Toma L. Olanzapine in severe Gilles de la Tourette syndrome: a 52-week doubleblind cross-over study vs low-dose pimozide. J Neurol. 2000; 247:443-446.

55. Müller-Vahl KR, Schneider U, Koblenz A, Jöbges M, Kolbe H, Daldrup T, Emrich HM. Treatment of Tourette's syndrome with $\Delta 9$-tetrahydrocannabinol (THC): a randomized crossover trial. Pharmacopsychiatry. 2002; 35:57-61.

56. Gadow KD, Sverd J, Sprafkin J, Nolan EE, Ezor SN. Efficacy of methylphenidate for attention-deficit hyperactivity disorder in children with tic disorder. Arch Gen Psychiatry. 1995; 52:444-455.

57. Gadow KD, Sverd J, Sprafkin J, Nolan EE, Grossman S. Long-term methylphenidate therapy in children with comorbid attention-deficit hyperactivity disorder and chronic multiple tic disorder. Arch Gen Psychiatry. 1999; 56: $330-336$

58. Gadow KD, Sverd J, Nolan EE, Sprafkin J, Schneider J. Immediate-release methylphenidate for ADHD in children with comorbid chronic multiple tic disorder. J Am Acad Child Adolesc Psychiatry. 2007; 46:840-848.

59. Castellanos FX, Giedd JN, Elia J, Marsh WL, Ritchie GF, Hamburger SD, Rapoport JL. Controlled stimulant treatment of ADHD and comorbid Tourette's syndrome: effects of stimulant and dose. J Am Acad Child Adolesc Psychiatry. 1997; 36:589-596.

60. Kurlan R, and Tourette's Syndrome Study Group. Treatment of ADHD in children with tics: a randomized controlled trial. Neurology. 2002; 58:527-36.

61. Silver AA, Shytle RD, Sheehan KH, Sheehan DV, Ramos A, Sanberg PR. Multicenter, double-blind, placebocontrolled study of mecamylamine monotherapy for Tourette's disorder. J Am Acad Child Adolesc Psychiatry. 2001; 40:1103-1110.

62. Gilbert DL, Dure L, Sethuraman G, Raab D, Lane J, Sallee FR. Tic reduction with pergolide in a randomized controlled trial in children. Neurology. 2003; 60:606-611.

63. Gilbert DL, Sethuraman G, Sine L, Peters S, Sallee FR. Tourette's syndrome improvement with pergolide in a randomized, double-blind, crossover trial. Neurology. 2000; 54:1310-1315.

64. Kurlan R, Crespi G, Coffey B, Mueller-Vahl K, Koval S, Wunderlich G, and Pramipexole for TS Trial Investigators. A multicenter randomized placebo-controlled clinical trial of pramipexole for Tourette's syndrome. Mov Disord. 2012; 27:775-78.

65. Goetz CG, Stebbins GT, Thelen JA. Talipexole and adult Gilles de la tourette's syndrome: Double-blind, placebo controlled clinical trial. Mov Disord. 1994; 9:315-317.

66. Singer HS, Wendlandt J, Krieger M, Giuliano J. Baclofen treatment in Tourette syndrome A double-blind, placebocontrolled, crossover trial. Neurology. 2001; 56:599-604.

67. Lemmon ME, Grados M, Kline T, Thompson CB, Ali SF, Singer HS. Efficacy of glutamate modulators in tic suppression: a double-blind, randomized control trial of D-serine and riluzole in Tourette syndrome. Pediatr Neurol. 2015; 52:629-634.

68. Bloch MH, Panza KE, Yaffa A, Alvarenga PG, Jakubovski E, Mulqueen JM, Landeros-Weisenberger A, Leckman JF. N-Acetylcysteine in the Treatment of Pediatric Tourette Syndrome: Randomized, Double-Blind, Placebo-Controlled 
Add-On Trial. J Child Adolesc Psychopharmacol. 2016; 26:327-34.

69. Allen AJ, Kurlan RM, Gilbert DL, Coffey BJ, Linder SL, Lewis DW, Winner PK, Dunn DW, Dure LS, Sallee FR, Milton DR, Mintz MI, Ricardi RK, et al. Atomoxetine treatment in children and adolescents with ADHD and comorbid tic disorders. Neurology. 2005; 65:1941-1949.

70. Spencer TJ, Sallee FR, Gilbert DL, Dunn DW, McCracken JT, Coffey BJ, Budman CL, Ricardi RK, Leonard HL, Allen AJ, Milton DR, Feldman PD, Kelsey DK, et al. Atomoxetine treatment of ADHD in children with comorbid Tourette syndrome. J Atten Disord. 2008; 11:470-481.

71. Silver AA, Shytle D, Philipp MK, Wilkinson BJ, McConville B, Sanberg PR. Transdermal nicotine and haloperidol in Tourette's disorder: a double-blind placebocontrolled study. J Clin Psychiatry. 2001; 62:707-714.

72. McConville BJ, Sanberg PR, Fogelson MH, King J, Cirino P, Parker KW, Norman AB. The effects of nicotine plus haloperidol compared to nicotine only and placebo nicotine only in reducing tic severity and frequency in Tourette's disorder. Biol Psychiatry. 1992; 31:832-840.

73. Zhao L, Li AY, Lv H, Liu FY, Qi FH. Traditional Chinese medicine Ningdong granule: the beneficial effects in Tourette's disorder. J Int Med Res. 2010; 38:169-175.

74. Zheng Y, Zhang ZJ, Han XM, Ding Y, Chen YY, Wang XF, Wei XW, Wang MJ, Cheng Y, Nie ZH, Zhao M, Zheng XX. A proprietary herbal medicine (5-Ling Granule) for Tourette syndrome: a randomized controlled trial. J Child Psychol Psychiatry. 2015; 57:74-83.

75. Li A, Cong S, Lu H, Li JJ, Zhao L. Clinical observation on treatment of Tourette syndrome by integrative medicine. Chin J Integr Med. 2009; 15:261-265.

76. Wu M, Xiao G, Zhang JM, Zhang X, Ma B, Wang SX, Zhou YB, Zhang JY. Clinical research into Qufeng Zhidong Recipe used to treat 31 children with tic disorder. J Tradit Chin Med. 2010; 30:163-170.

77. Wu M, Xiao GH, Yao M, Zhang JM, Zhang X, Zhou YB, Zhan g JY, Wang SX, Ma B, Chen YP. Multicenter clinical study on the treatment of children's tic disorder with Qufeng Zhidong Recipe. Chin J Integr Med. 2009; 15:254-60.

78. Toren P, Weizman A, Ratner S, Cohen D, Laor N. Ondansetron treatment in Tourette's disorder: a 3-week, randomized, double-blind, placebo-controlled study. J Clin Psychiatry. 2005; 66:499-503.

79. Nicolson R, Craven-Thuss B, Smith J, McKinlay BD, Castellanos FX. A randomized, double-blind, placebocontrolled trial of metoclopramide for the treatment of Tourette's disorder. J Am Acad Child Adolesc Psychiatry. 2005; 44:640-46.

80. Cohen SC, Mulqueen JM, Ferracioli-Oda E, Stuckelman ZD, Coughlin CG, Leckman JF, Bloch MH. Metaanalysis: Risk of tics associated With psychostimulan use in randomized, placebo-controlled trials. J Am Acad Child Adolesc Psychiatry. 2015; 54:728-736.

81. Li JJ. [An-yuan Li's experience of treatment for Tourette's disorder]. [Article in Chinese]. J Tradit Chin Med. 2009; 50:15-16.

82. Li AY, Ma RP, Lv H, Zhao L, Mu LM, Ma HB. [Clinical study of Ningdong granule in treating Tourette syndrome]. [Article in Chinese]. J Shandong Univ TCM. 2008; 32:33-35.

83. Lv H, Li A, Ma H, Liu F, Xu H. Effects of Ningdong granule on the dopamine system of Tourette's syndrome rat models. J Ethnopharmacol. 2009; 124:488-492.

84. Zhou SP, Song ZH, Zhang LL, Zhu YH, Cai N. Traditional Chinese medicinal composition for treating Tourette syndrome and preparation method of traditional Chinese medicinal composition. China Patent. 2011; CN102579842A. http://www.google.com/patents/CN102 $579842 \mathrm{~A}$ ?cl=zh.

85. Whittington $\mathrm{C}$, Pennant $\mathrm{M}$, Kendall $\mathrm{T}$, Glazebrook $\mathrm{C}$, Trayner P, Groom M, Hedderly T, Heyman I, Jackson G, Jackson S, Murphy T, Rickards H, Robertson M, et al. Practitioner Review: treatments for Tourette syndrome in children and young people - a systematic review. J Child Psychol Psychiatry. 2016; 57:988-1004.

86. Hollis C, Pennant M, Cuenca J, Glazebrook C, Kendall T, Whittington C, Stockton S, Larsson L, Bunton P, Dobson S, Groom M, Hedderly T, Heyman I, et al. Clinical effectiveness and patient perspectives of different treatment strategies for tics in children and adolescents with Tourette syndrome: a systematic review and qualitative analysis. Health Technol Assess. 2016; 20:1-450.

87. Weisman H, Qureshi IA, Leckman JF, Scahill L, Bloch MH. Systematic review: Pharmacological treatment of tic disorders - Efficacy of antipsychotic and alpha-2 adrenergic agonist agents. Neurosci Biobehav Rev. 2013; 37:1162-1171.

88. Moher D, Liberati A, Tetzlaff J, Altman DG, and PRISMA Group. Preferred reporting items for systematic reviews and meta-analyses: the PRISMA statement. Ann Intern Med. 2009; 151:264-69, W64.

89. Day S, Fayers P, Harvey D. Double data entry: what value, what price? Control Cl in Trials. 1998; 19:15-24.

90. Liberati A, Altman DG, Tetzlaff J, Mulrow C, Gotzsche PC, Ioannidis JP, Clarke M, Devereaux PJ, Kleijnen J, Moher D. The PRISMA statement for reporting systematic reviews and meta-analyses of studies that evaluate health careinterventions: explanation and elaboration. PLoS Med. 2009; 6:e1000100.

91. Leckman JF, Riddle MA, Hardin MT, Ort SI, Swartz KL, Stevenson J, Cohen DJ. The Yale Global Tic Severity Scale: Initial Testing of a Clinician-Rated Scale of Tic Severity. J Am Acad Child Adolesc Psychiatry. 1989; 28:566-73. 
92. Leckrnan JF, Towbin KE, Orr SI, Cohen DJ. Clinical assessment of tic disorder severity. New York:Wiley. 1988:56-58

93. Harcherik DF, Leckman JF, Detlor J, Cohen DJ. A New Instrument for Clinical Studies of Tourette's Syndrome. J Am Acad Child Psychiatry. 1984; 23:153-60.

94. Robertson MM, Scull DA, Eapen V, Trimble MR. Risperidone in the treatment of Tourette syndrome: a retrospective case note study. J Psychopharmacol. 1996; 10:317-20.

95. Higgins JPT, Altman DG. Chapter 8: Assessing risk of bias in included studies. Cochrane Handbook for Systematic Reviews of Interventions. The Cochrane Collaboration. 2011. https://doi.org/10.1002/9780470712184.ch8

96. Egger M, Davey Smith G, Schneider M, Minder C. Bias in meta-analysis detected by a simple, graphical test. BMJ. 1997; 315:629-34. 